TAIWANESE JOURNAL OF MATHEMATICS

Vol. 17, No. 4, pp. 1321-1352, August 2013

DOI: $10.11650 /$ tjm.17.2013.2559

This paper is available online at http://journal.taiwanmathsoc.org.tw

\title{
EXTENDED GENERAL NONLINEAR QUASI-VARIATIONAL INEQUALITIES AND PROJECTION DYNAMICAL SYSTEMS
}

\author{
Qamrul Hasan Ansari, Javad Balooee and Jen-Chih Yao*
}

\begin{abstract}
The aim of this paper is to introduce and study a new class of the extended general nonlinear quasi-variational inequalities and a new class of the extended general Wiener-Hopf equations. The equivalence between the extended general nonlinear quasi-variational inequalities and the fixed point problems, and as well as the extended general Wiener-Hopf equations is established. Then by using these equivalences, we discuss the existence and uniqueness of a solution of the extended general nonlinear quasi-variational inequalities. Applying the equivalent alternative formulation and a nearly uniformly Lipschitzian mapping $S$, we define some new $p$-step projection iterative algorithms with mixed errors for finding an element of set of the fixed points of nearly uniformly Lipschitzian mapping $S$ which is also a unique solution of the extended general nonlinear quasi-variational inequalities. The convergence analysis of the suggested iterative schemes under some suitable conditions is studied. We also suggest and analyze a class of extended general projection dynamical systems associated with the extended general nonlinear quasi-variational inequalities. We show that the trajectory of the solution of the extended general projection dynamical system converges globally exponential to a unique solution of the extended general nonlinear quasi-variational inequalities. Results obtained in this paper may be viewed as an refinement and improvement of the previously known results.
\end{abstract}

\section{INTRODUCTION}

The theory of variational inequalities is a well established subject in the area of pure and applied mathematics. In the last three decades, it has been extensively studied

Received October 23, 2012, accepted January 4, 2013.

Communicated by Jong-Shenq Guo.

2010 Mathematics Subject Classification: Primary 49J40; Secondary 47J20, 47H05.

Key words and phrases:

The research part of the first was done during his visit to KFUPM, Dhahran, Saudi Arabia. He would like to thanks KFUPM for providing excellent research facilities during his stay in KFUPM. In this research, third author was partially supported by a research grant NSC 99-2221-E-037-007-MY3 of National Science Council of Taiwan.

Variational inequalities, Fixed point problems, Nearly uniformly Lipschitzian mappings, Extended general Wiener-Hopf equations, Dynamical systems, Projection operator.

*Corresponding author. 
in the literature because of its applications to optimization, game theory, mechanics and engineering sciences. The quasi-variational inequality is a generalized of variational inequality in which the underlying set depends on the solution itself, see, for example, [1]. It was shown by Bensoussan and Lions [2] that a class of impulse control problems can be formulated as a quasi-variational inequality problem. For recent work on the generalized variants of quasi-variational inequalities and their applications, see for example $[2,9,14,18]$ and the references therein.

In recent years, much attention has been paid to consider and analyze the projected dynamical systems associated with variational inequalities and nonlinear programming problems, in which the right-hand side of the ordinary differential equation is a projection operator. Such types of the projected dynamical systems were introduced and studied by Dupuis and Nagurney [4]. Projected dynamical systems are characterized by a discontinuous right-hand side. The innovative and novel feature of a projected dynamical system is that its set of stationary points corresponds to the set of solutions of the corresponding variational inequality problems. Hence, the equilibrium and nonlinear problems arising in various branches of pure and applied sciences, which can be formulated in the form of variational inequalities, can also be studied in the more general setting of the projected dynamical systems. It has been shown in $[3,4,5,12,15,24,25,26]$ that the dynamical systems are useful in developing efficient and powerful numerical technique for solving variational inequalities and related optimization problems. Xia and Wang [24, 25], Zhang and Nagurney [26] and Nagurney and Zhang [12] have studied the globally asymptotic stability of these projected dynamical systems.

Very recently, Noor [14] introduced and studied a new class of quasi-variational inequalities, so called the general quasi-variational inequalities and proved that the class of general quasi-variational inequalities is equivalent to the fixed point problems and the Wiener-Hopf equations using the projection technique. Then, by using this equivalent alternative formulation, he suggested and analyzed some iterative methods for solving quasi-variational inequalities. He also studied the convergence analysis of the proposed iterative algorithms under some suitable conditions.

On the other hand, related to the variational inequalities, we have the problem of finding the fixed points of the nonexpansive mappings, which is the subject of current interest in functional analysis. It is natural to consider a unified approach to these two different problems. Motivated and inspired by the research going in this direction, Noor and Huang [16] considered the problem of finding the common element of the set of the solutions of variational inequalities and the set of the fixed points of the nonexpansive mappings. Noor [13] suggested and analyzed three-step iterative algorithms for finding the common elements of the set of the solutions of a variational inequality and the set of the fixed points of nonexpansive mappings. He also studied the convergence analysis of the suggested iterative algorithms under some suitable conditions. 
It is well known that every nonexpansive mapping is a Lipschitzian mapping. Lipschitzian mappings have been generalized by various authors. Sahu [19] introduced and investigated nearly uniformly Lipschitzian mappings as a generalization of Lipschitzian mappings.

Motivated and inspired by the recent research work going on, in this paper, we introduce and study a new class of the extended general nonlinear quasi-variational inequalities and a new class of the extended general Wiener-Hopf equations. We establish the equivalence between the extended general nonlinear quasi-variational inequalities and the fixed point problems, and as well as the extended general Wiener-Hopf equations. Then by using these equivalences, we discuss the existence and uniqueness of a solution of the extended general nonlinear quasi-variational inequalities. Applying the equivalent alternative formulation and a nearly uniformly Lipschitzian mapping $S$, we define a new $p$-step projection iterative algorithm with mixed errors for finding an element of the set of fixed points of nearly uniformly Lipschitzian mapping $S$ which is a unique solution of the extended general nonlinear quasi-variational inequalities. The convergence analysis of the suggested iterative schemes under some suitable conditions is studied. We also suggest and analyze a class of extended general projection dynamical systems associated with the extended general nonlinear quasi-variational inequalities. We show that the trajectory of the solution of the extended general projection dynamical system converges globally exponential to a unique solution of an extended general nonlinear quasi-variational inequality. Results obtained in this paper may be viewed as an refinement and improvement of the previously known results.

\section{Preliminaries and Basic Results}

Throughout this article, we assume that $\mathcal{H}$ is a real Hilbert space whose inner product and norm are denoted by $\langle.,$.$\rangle and \|$.$\| , respectively. Let K: \mathcal{H} \rightarrow 2^{\mathcal{H}}$ be a set-valued mapping such that for each $u \in \mathcal{H}, K(u)$ is a nonempty, closed and convex set.

For given nonlinear operators $T, g, h: \mathcal{H} \rightarrow \mathcal{H}$, we consider the problem of finding $u \in \mathcal{H}$ such that $h(u) \in K(u)$ and

$$
\langle\rho T(u)+h(u)-g(u), v-h(u)\rangle \geq 0, \quad \text { for all } v \in K(u),
$$

where $\rho>0$ is a constant. Inequality (2.1) is called the extended general nonlinear quasi-variational inequality (EGNQVI).

If $K(u) \equiv K$, the closed and convex set in $\mathcal{H}$, then the problem (2.1) is equivalent to the problem of finding $u \in \mathcal{H}$ such that $h(u) \in K$ and

$$
\langle\rho T(u)+h(u)-g(u), v-h(u)\rangle \geq 0, \quad \text { for all } v \in K,
$$

which is called the extended general nonlinear variational inequality and appears to be a new one. 
By taking different choices of the operators $g$ and $h$ in the above problems, one can easily obtain the problems studied in $[9,14]$ and the references therein.

We now recall the following well-known result and concepts.

Lemma 2.1. Let $K(u)$ be a nonempty closed and convex set in $\mathcal{H}$. Then, for a given $z \in \mathcal{H}, u \in K(u)$ satisfies the inequality

$$
\langle u-z, v-u\rangle \geq 0, \quad \text { for all } v \in K(u),
$$

if and only if $u=P_{K(u)} z$, where $P_{K(u)}$ is the projection of $\mathcal{H}$ onto the closed convex set $K(u)$.

It is well known that the projection operator $P_{K(u)}$ is nonexpansive, that is,

$$
\left\|P_{K(u)}(x)-P_{K(u)}(y)\right\| \leq\|x-y\|, \quad \text { for all } x, y \in \mathcal{H} .
$$

Definition 2.1. Let $T, g: \mathcal{H} \rightarrow \mathcal{H}$ be two single-valued operators. Then the operator $T$ is called

(a) monotone if

$$
\langle T(x)-T(y), x-y\rangle \geq 0, \quad \text { for all } x, y \in \mathcal{H}
$$

(b) $r$-strongly monotone if there exists a constant $r>0$ such that

$$
\langle T(x)-T(y), x-y\rangle \geq r\|x-y\|^{2}, \quad \text { for all } x, y \in \mathcal{H} ;
$$

(c) $\kappa$-strongly monotone with respect to $g$ if there exists a constant $\kappa>0$ such that

$$
\langle T(x)-T(y), g(x)-g(y)\rangle \geq \kappa\|g(x)-g(y)\|^{2}, \quad \text { for all } x, y \in \mathcal{H} ;
$$

(d) $\gamma$-Lipschitz continuous if there exists a constant $\gamma>0$ such that

$$
\|T(x)-T(y)\| \leq \gamma\|x-y\|, \quad \text { for all } x, y \in \mathcal{H} .
$$

It is also known as $\gamma$-Lipschitzian. If $\gamma=1$, then $T$ is called nonexpansive.

In the next definitions, several generalizations of the nonexpansive mappings which have been introduced by various authors in recent years are stated.

Definition 2.2. A nonlinear mapping $T: \mathcal{H} \rightarrow \mathcal{H}$ is called

(a) generalized Lipschitzian if there exists a constant $L>0$ such that

$$
\|T x-T y\| \leq L(\|x-y\|+1), \quad \text { for all } x, y \in \mathcal{H} ;
$$


(b) generalized ( $L, M)$-Lipschitzian [19] if there exist two constants $L, M>0$ such that

$$
\|T x-T y\| \leq L(\|x-y\|+M), \quad \text { for all } x, y \in \mathcal{H}
$$

(c) asymptotically nonexpansive [6] if there exists a sequence $\left\{k_{n}\right\} \subseteq[1, \infty)$ with $\lim _{n \rightarrow \infty} k_{n}=1$ such that for each $n \in \mathbb{N}$,

$$
\left\|T^{n} x-T^{n} y\right\| \leq k_{n}\|x-y\|, \quad \text { for all } x, y \in \mathcal{H} ;
$$

(d) pointwise asymptotically nonexpansive [8] if for each integer $n \geq 1$,

$$
\left\|T^{n} x-T^{n} y\right\| \leq \alpha_{n}(x)\|x-y\|, \quad \text { for all } x, y \in \mathcal{H},
$$

where $\alpha_{n}(x) \rightarrow 1$ pointwise on $X$;

(e) uniformly L-Lipschitzian if there exists a constant $L>0$ such that for each $n \in \mathbb{N}$,

$$
\left\|T^{n} x-T^{n} y\right\| \leq L\|x-y\|, \quad \text { for all } x, y \in \mathcal{H} .
$$

Definition 2.3. [19]. A nonlinear mapping $T: \mathcal{H} \rightarrow \mathcal{H}$ is said to be nearly Lipschitzian with respect to the sequence $\left\{a_{n}\right\}$ if for each $n \in \mathbb{N}$, there exists a constant $k_{n}>0$ such that

$$
\left\|T^{n} x-T^{n} y\right\| \leq k_{n}\left(\|x-y\|+a_{n}\right), \quad \text { for all } x, y \in \mathcal{H},
$$

where $\left\{a_{n}\right\}$ is a fix sequence in $[0, \infty)$ with $a_{n} \rightarrow 0$, as $n \rightarrow \infty$.

For an arbitrary, but fixed $n \in \mathbb{N}$, the infimum of constants $k_{n}$ in (2.3) is called nearly Lipschitz constant and is denoted by $\eta\left(T^{n}\right)$. Notice that

$$
\eta\left(T^{n}\right)=\sup \left\{\frac{\left\|T^{n} x-T^{n} y\right\|}{\|x-y\|+a_{n}}: x, y \in \mathcal{H}, x \neq y\right\} .
$$

Definition 2.4. A nearly Lipschitzian mapping $T: \mathcal{H} \rightarrow \mathcal{H}$ with the sequence $\left\{\left(a_{n}, \eta\left(T^{n}\right)\right)\right\}$ is said to be

(a) nearly nonexpansive if $\eta\left(T^{n}\right)=1$ for all $n \in \mathbb{N}$, that is,

$$
\left\|T^{n} x-T^{n} y\right\| \leq\|x-y\|+a_{n}, \quad \text { for all } x, y \in \mathcal{H} ;
$$

(b) nearly asymptotically nonexpansive if $\eta\left(T^{n}\right) \geq 1$ for all $n \in \mathbb{N}$ and $\lim _{n \rightarrow \infty} \eta\left(T^{n}\right)=$ 1 , that is, $k_{n} \geq 1$ for all $n \in \mathbb{N}$ with $\lim _{n \rightarrow \infty} k_{n}=1$;

(c) nearly uniformly L-Lipschitzian if $\eta\left(T^{n}\right) \leq L$ for all $n \in \mathbb{N}$, that is, $k_{n}=L$ for all $n \in \mathbb{N}$. 
Remark 2.2. (a) Every nonexpansive mapping is a asymptotically nonexpansive mapping, and every asymptotically nonexpansive mapping is a pointwise asymptotically nonexpansive mapping. Also, the class of Lipschitzian mappings properly includes the class of pointwise asymptotically nonexpansive mappings.

(b) It is obvious that every Lipschitzian mapping is a generalized Lipschitzian mapping. Furthermore, every mapping with a bounded range is a generalized Lipschitzian mapping. It is easy to see that the class of generalized $(L, M)$ Lipschitzian mappings is more general than the class of generalized Lipschitzian mappings.

(c) Clearly, the class of nearly uniformly $L$-Lipschitzian mappings properly includes the class of generalized $(L, M)$-Lipschitzian mappings and that of uniformly $L$-Lipschitzian mappings. Note that every nearly asymptotically nonexpansive mapping is nearly uniformly $L$-Lipschitzian.

We present some new examples to investigate relations between these mappings.

Example 2.3. Let $\mathcal{H}=\mathbb{R}$ and define $T: \mathcal{H} \rightarrow \mathcal{H}$ by

$$
T(x)= \begin{cases}\frac{1}{\gamma}, & \text { if } x \in[0, \gamma], \\ 0, & \text { if } x \in(-\infty, 0) \cup(\gamma, \infty),\end{cases}
$$

where $\gamma>1$ is a constant. Evidently, the mapping $T$ is discontinuous at the points $x=0, \gamma$. Since every Lipschitzian mapping is continuous, it follows that $T$ is not Lipschitzian. For each $n \in \mathbb{N}$, take $a_{n}=\frac{1}{\gamma^{n}}$. Then

$$
|T x-T y| \leq|x-y|+\frac{1}{\gamma}=|x-y|+a_{1}, \quad \text { for all } x, y \in \mathbb{R} .
$$

Since $T^{n} z=\frac{1}{\gamma}$, for all $z \in \mathbb{R}$ and $n \geq 2$, it follows that for all $x, y \in \mathbb{R}$ and $n \geq 2$,

$$
\left|T^{n} x-T^{n} y\right| \leq|x-y|+\frac{1}{\gamma^{n}}=|x-y|+a_{n} .
$$

Hence, $T$ is a nearly nonexpansive mapping with respect to the sequence $\left\{a_{n}\right\}=\left\{\frac{1}{\gamma^{n}}\right\}$.

The following example shows that the nearly uniformly $L$-Lipschitzian mappings are not necessarily continuous.

Example 2.4. Let $\mathcal{H}=[0, b]$, where $b \in(0,1]$, and let $T: \mathcal{H} \rightarrow \mathcal{H}$ be defined as

$$
T(x)=\left\{\begin{array}{cl}
\gamma x, & \text { if } x \in[0, b), \\
0, & \text { if } x=b,
\end{array}\right.
$$


where $\gamma \in(0,1)$. Then, clearly $T$ is discontinuous at $b$. Hence, $T$ is not a Lipschitzian mapping. Take for each $n \in \mathbb{N}, a_{n}=\gamma^{n-1}$. Then for all $n \in \mathbb{N}$ and $x, y \in[0, b)$, we have

$$
\begin{aligned}
\left|T^{n} x-T^{n} y\right| & =\left|\gamma^{n} x-\gamma^{n} y\right|=\gamma^{n}|x-y| \leq \gamma^{n}|x-y|+\gamma^{n} \\
& \leq \gamma|x-y|+\gamma^{n}=\gamma\left(|x-y|+a_{n}\right) .
\end{aligned}
$$

If $x \in[0, b)$ and $y=b$, then for each $n \in \mathbb{N}$, we have $T^{n} x=\gamma^{n} x$ and $T^{n} y=0$. Since $0<|x-y| \leq b \leq 1$, it follows that for all $n \in \mathbb{N}$,

$$
\begin{aligned}
\left|T^{n} x-T^{n} y\right| & =\left|\gamma^{n} x-0\right|=\gamma^{n} x \leq \gamma^{n} b \leq \gamma^{n}<\gamma^{n}|x-y|+\gamma^{n} \\
& \leq \gamma|x-y|+\gamma^{n}=\gamma\left(|x-y|+a_{n}\right) .
\end{aligned}
$$

Hence, $T$ is a nearly uniformly $\gamma$-Lipschitzian mapping with respect to the sequence $\left\{a_{n}\right\}=\left\{\gamma^{n-1}\right\}$.

Obviously, every nearly nonexpansive mapping is a nearly uniformly Lipschitzian mapping. In the following example, we show that the class of nearly uniformly Lipschitzian mappings properly includes the class of nearly nonexpansive mappings.

Example 2.5. Let $\mathcal{H}=\mathbb{R}$ and let $T: \mathcal{H} \rightarrow \mathcal{H}$ be defined as

$$
T(x)= \begin{cases}\frac{1}{2}, & \text { if } x \in[0,1) \cup\{2\} \\ 2, & \text { if } x=1, \\ 0, & \text { if } x \in(-\infty, 0) \cup(1,2) \cup(2,+\infty) .\end{cases}
$$

Evidently, the mapping $T$ is discontinuous at $x=0,1,2$. Hence, $T$ is not a Lipschitzian mapping. Take for each $n \in \mathbb{N}, a_{n}=\frac{1}{2^{n}}$. Then $T$ is not a nearly nonexpansive mapping with respect to the sequence $\left\{\frac{1}{2^{n}}\right\}$, because taking $x=1$ and $y=\frac{1}{2}$, we have $T x=2$, $T y=\frac{1}{2}$ and

$$
|T x-T y|>|x-y|+\frac{1}{2}=|x-y|+a_{1}
$$

However,

$$
|T x-T y| \leq 4\left(|x-y|+\frac{1}{2}\right)=4\left(|x-y|+a_{1}\right), \quad \text { for all } x, y \in \mathbb{R}
$$

and for all $n \geq 2$,

$$
\left|T^{n} x-T^{n} y\right| \leq 4\left(|x-y|+\frac{1}{2^{n}}\right)=4\left(|x-y|+a_{n}\right), \quad \text { for all } x, y \in \mathbb{R},
$$

since $T^{n} z=\frac{1}{2}$, for all $z \in \mathbb{R}$ and $n \geq 2$. Hence, for each $L \geq 4, T$ is a nearly uniformly $L$-Lipschitzian mapping with respect to the sequence $\left\{\frac{1}{2^{n}}\right\}$. 
It is clear that every uniformly $L$-Lipschitzian mapping is a nearly uniformly $L$ Lipschitzian mapping. In the next example, we show that the class nearly uniformly $L$-Lipschitzian mappings properly includes the class of uniformly $L$-Lipschitzian mappings.

Example 2.6. Let $\mathcal{H}=\mathbb{R}$ and let the self-mapping $T$ of $\mathcal{H}$ be defined as in Example 2.5. Then $T$ is not a uniformly 4-Lipschitzian mapping. Since if $x=1$ and $y \in\left(1, \frac{3}{2}\right)$, then we have $|T x-T y|>4|x-y|$, because $0<|x-y|<\frac{1}{2}$. But, in view of Example 2.5, $T$ is a nearly uniformly 4-Lipschitzian mapping.

An example can be easily constructed to show that the class of generalized Lipschitzian mappings properly includes the class of Lipschitzian mappings and that of mappings with bounded range.

\section{EXISTENCE AND Uniqueness of a SOlution}

In this section, we prove the existence and uniqueness theorem for a solution of extended general nonlinear quasi-variational inequality (2.1). We establish the equivalence between problem (2.1) and a fixed point problem using Lemma 2.1.

Lemma 3.1. Let $T, g, h$ and $\rho$ be the same as in problem (2.1). Then $u \in \mathcal{H}$ with $h(u) \in K(u)$ is a solution of problem (2.1) if and only if

$$
h(u)=P_{K(u)}(g(u)-\rho T(u)) .
$$

Proof. Let $u \in \mathcal{H}$ with $h(u) \in K(u)$ be a solution of problem (2.1). Then, by using (2.1), we have

$$
\langle h(u)-(g(u)-\rho T(u)), v-h(u)\rangle \geq 0, \quad \text { for all } v \in K(u)
$$

which in view of Lemma 2.1 is equivalent to

$$
h(u)=P_{K(u)}(g(u)-\rho T(u)) .
$$

By using above lemma, we prove the existence of a unique solution of extended general nonlinear quasi-variational inequality (2.1).

Theorem 3.2. Let $T, g, h$ and $\rho$ be the same as in problem (2.1). Suppose that $T$ is $\delta$-strongly monotone with respect to $g$ and $\sigma$-Lipschitz continuous, $h$ is $\varrho$-strongly monotone and $\nu$-Lipschitz continuous, and $g$ is $\tau$-Lipschitz continuous. Further, let there exists a constant $\varsigma>0$ such that

$$
\left\|P_{K(u)}(w)-P_{K(v)}(w)\right\| \leq \varsigma\|u-v\|, \quad \text { for all } u, v, w \in \mathcal{H} .
$$


If the constant $\rho$ satisfies the following conditions

$$
\left\{\begin{array}{l}
\left|\rho-\frac{\delta \tau^{2}}{\sigma^{2}}\right|<\frac{\sqrt{\delta^{2} \tau^{4}-\sigma^{2}\left(\tau^{2}-(1-\mu)^{2}\right)}}{\sigma^{2}} \\
\delta \tau^{2}>\sigma \sqrt{\tau^{2}-(1-\mu)^{2}} \\
\mu=\varsigma+\sqrt{1-\left(2 \varrho-\nu^{2}\right)}<1 \\
2 \varrho<1+\nu^{2}, \quad \tau+\mu>1
\end{array}\right.
$$

then problem (2.1) admits a unique solution.

Proof. Define the mapping $\psi: \mathcal{H} \rightarrow \mathcal{H}$ by

$$
\psi(x)=x-h(x)+P_{K(x)}(g(x)-\rho T(x)), \quad \text { for all } x \in \mathcal{H} .
$$

We claim that $\psi$ is a contraction mapping. For this end, let $x, \hat{x} \in \mathcal{H}$ be given. It follows from (3.4) and the condition (3.2) that

$$
\begin{aligned}
& \|\psi(x)-\psi(\hat{x})\| \\
\leq & \|x-\hat{x}-(h(x)-h(\hat{x}))\|+\left\|P_{K(x)}(g(x)-\rho T(x))-P_{K(\hat{x})}(g(\hat{x})-\rho T(\hat{x}))\right\| \\
\leq & \|x-\hat{x}-(h(x)-h(\hat{x}))\|+\left\|P_{K(x)}(g(x)-\rho T(x))-P_{K(\hat{x})}(g(x)-\rho T(x))\right\| \\
& +\left\|P_{K(\hat{x})}(g(x)-\rho T(x))-P_{K(\hat{x})}(g(\hat{x})-\rho T(\hat{x}))\right\| \\
\leq & \|x-\hat{x}-(h(x)-h(\hat{x}))\|+\varsigma\|x-\hat{x}\|+\|g(x)-g(\hat{x})-\rho(T(x)-T(\hat{x}))\| .
\end{aligned}
$$

From $\varrho$-strongly monotonicity and $\nu$-lipschitz continuity of $h$, it follows that

$$
\begin{aligned}
& \|x-\hat{x}-(h(x)-h(\hat{x}))\|^{2} \\
= & \|x-\hat{x}\|^{2}-2\langle h(x)-h(\hat{x}), x-\hat{x}\rangle+\|h(x)-h(\hat{x})\|^{2} \\
\leq & (1-2 \varrho)\|x-\hat{x}\|^{2}+\|h(x)-h(\hat{x})\|^{2} \\
\leq & \left(1-2 \varrho+\nu^{2}\right)\|x-\hat{x}\|^{2} .
\end{aligned}
$$

Since $T$ is $\delta$-strongly monotone with respect to $g$ and $\sigma$-Lipschitz continuous, and $g$ is $\tau$-Lipschitz continuous, we obtain

$$
\begin{aligned}
& \|g(x)-g(\hat{x})-\rho(T(x)-T(\hat{x}))\|^{2} \\
= & \|g(x)-g(\hat{x})\|^{2}-2 \rho\langle T(x)-T(\hat{x}), g(x)-g(\hat{x})\rangle+\rho^{2}\|T(x)-T(\hat{x})\|^{2} \\
\leq & (1-2 \rho \delta)\|g(x)-g(\hat{x})\|^{2}+\rho^{2}\|T(x)-T(\hat{x})\|^{2} \\
\leq & \left((1-2 \rho \delta) \tau^{2}+\rho^{2} \sigma^{2}\right)\|x-\hat{x}\|^{2} .
\end{aligned}
$$

Substituting (3.6) and (3.7) in (3.5), we get

$$
\|\psi(x)-\psi(\hat{x})\| \leq \omega\|x-\hat{x}\|,
$$


where

$$
\omega=\varsigma+\sqrt{1-2 \varrho+\nu^{2}}+\sqrt{(1-2 \rho \delta) \tau^{2}+\rho^{2} \sigma^{2}} .
$$

The condition (3.3) implies that $0 \leq \omega<1$, and so, from (3.8) we conclude that the mapping $\psi$ is contraction. According to Banach fixed point theorem, $\psi$ has a unique fixed point in $\mathcal{H}$, that is, there exists a unique point $u \in \mathcal{H}$ such that $\psi(u)=u$. It follows from (3.4) that $h(u)=P_{K(u)}(g(u)-\rho T(u))$. Lemma 3.1 guarantees that $u \in \mathcal{H}$ with $h(u) \in K(u)$ is a unique solution of problem (2.1).

Remark 3.3. Theorem 3.2 improves and extends Theorem 3.1 in [14].

Remark 3.4. [14]. In many important applications [7, 9, 21, 22], the convexvalued set $K(u)$ can be considered as

$$
K(u)=m(u)+K,
$$

where $m(u)$ is a point-to-point mapping and $K$ is a convex set. Then, we have

$$
P_{K(u)}(w)=P_{m(u)+K}(w)=m(u)+P_{K}(w-m(u)), \quad \text { for all } u, w \in \mathcal{H} .
$$

We not that if $K(u)$ is defined by (3.10) and $m(u)$ is a Lipschitz continuous mapping with constant $\gamma>0$, then

$$
\begin{aligned}
\left\|P_{K(u)}(w)-P_{K(v)}(w)\right\| & =\left\|m(u)-m(v)+P_{K}(w-m(u))-P_{K}(w-m(v))\right\| \\
& \leq 2\|m(u)-m(v)\| \leq 2 \gamma\|u-v\|, \quad \text { for all } u, v, w \in \mathcal{H}
\end{aligned}
$$

which shows that the condition (3.2) holds with $\varsigma=2 \gamma$.

\section{Projection Algorithms and Convergence Analysis}

In this section, we suggest and analyze some new finite step projection iterative algorithms with mixed errors for finding a fixed point of nearly uniformly Lipschitzian mapping $S$ which is also a unique solution of the extended general nonlinear quasivariational inequality (2.1). Furthermore, the convergence analysis of the suggested iterative algorithms under some suitable conditions is studied.

Let $S: \mathcal{H} \rightarrow \mathcal{H}$ be a nearly uniformly Lipschitzian mapping. We denote the set of all the fixed points of $S$ by $\operatorname{Fix}(S)$, and the set of all the solutions of problem (2.1) by $\operatorname{EGNQVI}(\mathcal{H}, T, g, h)$. If $u \in \operatorname{Fix}(S) \cap \operatorname{EGNQVI}(\mathcal{H}, T, g, h)$, then it follows from Lemma 3.1 that for each $n \geq 0$,

$$
\begin{aligned}
u & =S^{n} u=u-h(u)+P_{K(u)}(g(u)-\rho T(u)) \\
& =S^{n}\left[u-h(u)+P_{K(u)}(g(u)-\rho T(u))\right] .
\end{aligned}
$$


The fixed point formulation (4.1) enables us to define the following $p$-step projection iterative algorithm with mixed errors for finding a fixed point of nearly uniformly Lipschitzian mapping $S$ which is also a solution of the extended general nonlinear quasi-variational inequality (2.1).

Algorithm 4.1. Let $T, g, h$ and $\rho$ be the same as in problem (2.1). For an arbitrary chosen initial point $u_{0} \in \mathcal{H}$, compute the iterative sequence $\left\{u_{n}\right\}_{n=0}^{\infty}$ in the following way:

$$
\left\{\begin{array}{l}
u_{n+1}=\left(1-\alpha_{n, 1}-\beta_{n, 1}\right) u_{n}+\alpha_{n, 1}\left(S^{n} \Psi\left(v_{n, 1}\right)+e_{n, 1}\right)+\beta_{n, 1} l_{n, 1}+r_{n, 1}, \\
v_{n, i}=\left(1-\alpha_{n, i+1}-\beta_{n, i+1}\right) u_{n}+\alpha_{n, i+1}\left(S^{n} \Psi\left(v_{n, i+1}\right)+e_{n, i+1}\right) \\
\quad+\beta_{n, i+1} l_{n, i+1}+r_{n, i+1} \\
\quad \vdots \\
v_{n, p-1}=\left(1-\alpha_{n, p}-\beta_{n, p}\right) u_{n}+\alpha_{n, p}\left(S^{n} \Psi\left(u_{n}\right)+e_{n, p}\right)+\beta_{n, p} l_{n, p}+r_{n, p} \\
i=1,2, \ldots, p-2
\end{array}\right.
$$

where

$$
\left\{\begin{array}{l}
\Psi\left(v_{n, i}\right)=v_{n, i}-h\left(v_{n, i}\right)+P_{K\left(v_{n, i}\right)}\left(g\left(v_{n, i}\right)-\rho T\left(v_{n, i}\right)\right), \\
\Psi\left(u_{n}\right)=u_{n}-h\left(u_{n}\right)+P_{K\left(u_{n}\right)}\left(g\left(u_{n}\right)-\rho T\left(u_{n}\right)\right) \\
i=1,2, \ldots, p-1
\end{array}\right.
$$

$S: \mathcal{H} \rightarrow \mathcal{H}$ is a nearly uniformly Lipschitzian mapping, $\left\{\alpha_{n, i}\right\}_{n=0}^{\infty},\left\{\beta_{n, i}\right\}_{n=0}^{\infty}(i=$ $1,2, \ldots, p)$ are $2 p$ sequences in $[0,1]$ such that $\sum_{n=0}^{\infty} \prod_{i=1}^{p} \alpha_{n, i}=\infty, \alpha_{n, i}+\beta_{n, i} \leq 1$, for all $n \geq 0$ and for each $i=1,2, \ldots, p, \sum_{n=0}^{\infty} \beta_{n, i}<\infty$ for each $i=1,2, \ldots, p$, and $\left\{e_{n, i}\right\}_{n=0}^{\infty},\left\{l_{n, i}\right\}_{n=0}^{\infty},\left\{r_{n, i}\right\}_{n=0}^{\infty}(i=1,2 \ldots p)$ are $3 p$ sequences in $\mathcal{H}$ to take into account a possible inexact computation of the resolvent operator point satisfying the following conditions: $\left\{l_{n, i}\right\}_{n=0}^{\infty}(i=1,2, \ldots, p)$ are $p$ bounded sequences in $\mathcal{H}$ and $\left\{e_{n, i}\right\}_{n=0}^{\infty},\left\{r_{n, i}\right\}_{n=0}^{\infty}$ are $2 p$ sequences in $\mathcal{H}$ such that

$$
\left\{\begin{array}{l}
e_{n, i}=e_{n, i}^{\prime}+e_{n, i}^{\prime \prime}, \quad n \geq 0, i=1,2, \ldots, p, \\
\lim _{n \rightarrow \infty}\left\|e_{n, i}^{\prime}\right\|=0, \quad i=1,2, \ldots, p, \\
\sum_{n=0}^{\infty}\left\|e_{n, i}^{\prime \prime}\right\|<\infty, \quad \sum_{n=0}^{\infty}\left\|r_{n, i}\right\|<\infty, \quad i=1,2, \ldots, p .
\end{array}\right.
$$


Algorithm 4.2. Let $T, g, h$ and $\rho$ be the same as in Algorithm 4.1. For an arbitrary chosen initial point $u_{0} \in \mathcal{H}$, compute the iterative sequence $\left\{u_{n}\right\}_{n=0}^{\infty}$ by the following iterative process:

$$
u_{n+1}=\left(1-\alpha_{n}\right) u_{n}+\alpha_{n} S^{n}\left\{u_{n}-h\left(u_{n}\right)+P_{K\left(u_{n}\right)}\left(g\left(u_{n}\right)-\rho T\left(u_{n}\right)\right)\right\},
$$

where $S$ is the same as in Algorithm 4.1 and $\left\{\alpha_{n}\right\}_{n=0}^{\infty}$ is a sequence in $[0,1]$ with $\sum_{n=0}^{\infty} \alpha_{n}=\infty$.

If $S \equiv I$, then Algorithms 4.1 and 4.2 reduce to the following algorithms, respectively.

Algorithm 4.3. Assume that $T, g, h$ and $\rho$ are the same as in Algorithm 4.1. For an arbitrary chosen initial point $u_{0} \in \mathcal{H}$, compute the iterative sequence $\left\{u_{n}\right\}_{n=0}^{\infty}$ by the following iterative processes:

$$
\left\{\begin{array}{l}
u_{n+1}=\left(1-\alpha_{n, 1}-\beta_{n, 1}\right) u_{n}+\alpha_{n, 1}\left(\Psi\left(v_{n, 1}\right)+e_{n, 1}\right)+\beta_{n, 1} l_{n, 1}+r_{n, 1}, \\
v_{n, i}=\left(1-\alpha_{n, i+1}-\beta_{n, i+1}\right) u_{n}+\alpha_{n, i+1}\left(\Psi\left(v_{n, i+1}\right)+e_{n, i+1}\right)+\beta_{n, i+1} l_{n, i+1}+r_{n, i+1}, \\
\quad \vdots \\
v_{n, p-1}=\left(1-\alpha_{n, p}-\beta_{n, p}\right) u_{n}+\alpha_{n, p}\left(\Psi\left(u_{n}\right)+e_{n, p}\right)+\beta_{n, p} l_{n, p}+r_{n, p}, \\
i=1,2, \ldots, p-2,
\end{array}\right.
$$

where $\Psi\left(v_{n, i}\right)(i=1,2, \ldots, p-1), \Psi\left(u_{n}\right),\left\{\alpha_{n, i}\right\}_{n=0}^{\infty},\left\{\beta_{n, i}\right\}_{n=0}^{\infty},\left\{e_{n, i}\right\}_{n=0}^{\infty},\left\{l_{n, i}\right\}_{n=0}^{\infty}$, $\left\{r_{n, i}\right\}_{n=0}^{\infty}(i=1,2, \ldots, p)$, are the same as in Algorithm 4.1.

Algorithm 4.4. Let $T, g, h$ and $\rho$ be the same as in Algorithm 4.1. For an arbitrary chosen initial point $u_{0} \in \mathcal{H}$, compute the iterative sequence $\left\{u_{n}\right\}_{n=0}^{\infty}$ by the following iterative process:

$$
u_{n+1}=\left(1-\alpha_{n}\right) u_{n}+\alpha_{n}\left\{u_{n}-h\left(u_{n}\right)+P_{K\left(u_{n}\right)}\left(g\left(u_{n}\right)-\rho T\left(u_{n}\right)\right)\right\},
$$

where the sequence $\left\{\alpha_{n}\right\}_{n=0}^{\infty}$ is the same as in Algorithm 4.2.

Remark 4.5. (a) If $e_{n, i}=r_{n, i}=0$ for all $n \geq 0$ and $i=1,2, \ldots p$, then Algorithms 4.1 and 4.3 reduce into the perturbed iterative processes with mean errors.

(b) When $e_{n, i}=l_{n, i}=r_{n, i}=0$, for all $n \geq 0$ and $i=1,2, \ldots p$, then Algorithms 4.1 and 4.3 become the perturbed iterative processes without error. 
Remark 4.6. Algorithms 4.1-4.3 in [14] are special cases of Algorithms 4.1-4.4. In brief, for a suitable and appropriate choice of the operators $T, g, h$ and the constant $\rho>0$, one can obtain a number of new and previously known iterative schemes for solving problem (2.1) and related problems. This clearly shows that Algorithms 4.1-4.4 are quite general and unifying.

We study the convergence analysis of the suggested iterative Algorithm 4.1 under some suitable conditions. For this end, we need the following lemma whose proof directly follows from Lemma 2 in Liu [10].

Lemma 4.7. Let $\left\{a_{n}\right\},\left\{b_{n}\right\}$ and $\left\{c_{n}\right\}$ be three nonnegative real sequences satisfying the following condition: There exists a natural number $n_{0}$ such that

$$
a_{n+1} \leq\left(1-t_{n}\right) a_{n}+b_{n} t_{n}+c_{n}, \quad \forall n \geq n_{0},
$$

where $t_{n} \in[0,1], \sum_{n=0}^{\infty} t_{n}=\infty, \lim _{n \rightarrow \infty} b_{n}=0, \sum_{n=0}^{\infty} c_{n}<\infty$.

Then $\lim _{n \rightarrow 0} a_{n}=0$.

Theorem 4.8. Let $T, g, h$ and $\rho$ be the same as in Theorem 3.2 and let all the conditions of Theorem 3.2 hold. Let $S: \mathcal{H} \rightarrow \mathcal{H}$ be a nearly uniformly L-Lipschitzian mapping with the sequence $\left\{b_{n}\right\}_{n=0}^{\infty}$ such that $\operatorname{Fix}(S) \cap \operatorname{EGNQVI}(\mathcal{H}, T, g, h) \neq \emptyset$. Further, let $L \omega<1$, where $\omega$ is the same as in (3.9). If there exists a constant $\alpha>0$ such that $\prod_{i=1}^{p} \alpha_{n, i} \geq \alpha$ for each $n \geq 0$, then the iterative sequence $\left\{u_{n}\right\}_{n=0}^{\infty}$ generated by Algorithm 4.1 converges strongly to a unique element $u^{*}$ of $\operatorname{Fix}(S) \cap$ $\operatorname{EGNQVI}(\mathcal{H}, T, g, h)$.

Proof. Theorem 3.2 guarantees the existence of a unique solution $u^{*} \in \mathcal{H}$ with $h\left(u^{*}\right) \in K\left(u^{*}\right)$ for problem (2.1). Hence, in view of Lemma 3.1, $h\left(u^{*}\right)=$ $P_{K\left(u^{*}\right)}\left(g\left(u^{*}\right)-\rho T\left(u^{*}\right)\right)$. Since $\operatorname{EGNQVI}(\mathcal{H}, T, g, h)$ is a singleton set, it follows from $\operatorname{Fix}(S) \cap \operatorname{EGNQVI}(\mathcal{H}, T, g, h) \neq \emptyset$ that $u^{*} \in \operatorname{Fix}(S)$. Consequently, for each $i \in\{1,2, \ldots, p\}$ and $n \geq 0$, we have

$$
\begin{aligned}
u^{*}= & \left(1-\alpha_{n, i}-\beta_{n, i}\right) u^{*}+\alpha_{n, i} S^{n}\left[u^{*}-h\left(u^{*}\right)\right. \\
& \left.+P_{K\left(u^{*}\right)}\left(g\left(u^{*}\right)-\rho T\left(u^{*}\right)\right)\right]+\beta_{n, i} u^{*},
\end{aligned}
$$

where the sequences $\left\{\alpha_{n, i}\right\}_{n=0}^{\infty}$ and $\left\{\beta_{n, i}\right\}_{n=0}^{\infty}(i=1,2, \ldots p)$ are the same as in Algorithm 4.1. Let $\Omega=\sup _{n \geq 0}\left\{\left\|l_{n, i}-u^{*}\right\|: i=1,2, \ldots, p\right\}$. By using (4.2), (4.4) and the condition (3.2), we have 


$$
\begin{aligned}
& \left\|u_{n+1}-u^{*}\right\| \\
\leq & \left(1-\alpha_{n, 1}-\beta_{n, 1}\right)\left\|u_{n}-u^{*}\right\|+\alpha_{n, 1} \| S^{n}\left\{v_{n, 1}-h\left(v_{n, 1}\right)\right. \\
& \left.+P_{K\left(v_{n, 1}\right)}\left(g\left(v_{n, 1}\right)-\rho T\left(v_{n, 1}\right)\right)\right\}-S^{n}\left\{u^{*}-h\left(u^{*}\right)\right. \\
& \left.+P_{K\left(u^{*}\right)}\left(g\left(u^{*}\right)-\rho T\left(u^{*}\right)\right)\right\}\left\|+\beta_{n, 1}\right\| l_{n, 1}-u^{*}\left\|+\alpha_{n, 1}\right\| e_{n, 1}\|+\| r_{n, 1} \| \\
& \left.+P_{K\left(u^{*}\right)}\left(g\left(u^{*}\right)-\rho T\left(u^{*}\right)\right)\right\}\left\|+\beta_{n, 1}\right\| l_{n, 1}-u^{*}\left\|+\alpha_{n, 1}\right\| e_{n, 1}\|+\| r_{n, 1} \| \\
\leq & \left(1-\alpha_{n, 1}-\beta_{n, 1}\right)\left\|u_{n}-u^{*}\right\|+\alpha_{n, 1} L\left(\left\|v_{n, 1}-u^{*}-\left(h\left(v_{n, 1}\right)-h\left(u^{*}\right)\right)\right\|\right. \\
& \left.+\left\|P_{K\left(v_{n, 1}\right)}\left(g\left(v_{n, 1}\right)-\rho T\left(v_{n, 1}\right)\right)-P_{K\left(u^{*}\right)}\left(g\left(u^{*}\right)-\rho T\left(u^{*}\right)\right)\right\|+b_{n}\right) \\
& +\alpha_{n, 1}\left(\left\|e_{n, 1}^{\prime}\right\|+\left\|e_{n, 1}^{\prime \prime}\right\|\right)+\left\|r_{n, 1}\right\|+\beta_{n, 1} \Omega \\
\leq & \left(1-\alpha_{n, 1}-\beta_{n, 1}\right)\left\|u_{n}-u^{*}\right\|+\alpha_{n, 1} L\left(\left\|v_{n, 1}-u^{*}-\left(h\left(v_{n, 1}\right)-h\left(u^{*}\right)\right)\right\|\right. \\
& +\left\|P_{K\left(v_{n, 1}\right)}\left(g\left(u^{*}\right)-\rho T\left(u^{*}\right)\right)-P_{K\left(u^{*}\right)}\left(g\left(u^{*}\right)-\rho T\left(u^{*}\right)\right)\right\| \\
& \left.+\left\|P_{K\left(v_{n, 1}\right)}\left(g\left(v_{n, 1}\right)-\rho T\left(v_{n, 1}\right)\right)-P_{K\left(v_{n, 1}\right)}\left(g\left(u^{*}\right)-\rho T\left(u^{*}\right)\right)\right\|+b_{n}\right) \\
& +\alpha_{n, 1}\left\|e_{n, 1}^{\prime}\right\|+\left\|e_{n, 1}^{\prime \prime}\right\|+\left\|r_{n, 1}\right\|+\beta_{n, 1} \Omega \\
\leq & \left(1-\alpha_{n, 1}-\beta_{n, 1}\right)\left\|u_{n}-u^{*}\right\|+\alpha_{n, 1} L\left(\left\|v_{n, 1}-u^{*}-\left(h\left(v_{n, 1}\right)-h\left(u^{*}\right)\right)\right\|\right. \\
& \left.+\varsigma\left\|v_{n, 1}-u^{*}\right\|+\left\|g\left(v_{n, 1}\right)-g\left(u^{*}\right)-\rho\left(T\left(v_{n, 1}\right)-T\left(u^{*}\right)\right)\right\|+b_{n}\right) \\
& +\alpha_{n, 1}\left\|e_{n, 1}^{\prime}\right\|+\left\|e_{n, 1}^{\prime \prime}\right\|+\left\|r_{n, 1}\right\|+\beta_{n, 1} \Omega .
\end{aligned}
$$

Since $T$ is $\delta$-strongly monotone with respect to $g$ and $\sigma$-Lipschitz continuous, $g$ is $\tau$ Lipschitz continuous, $h$ is $\varrho$-strongly monotone and $\nu$-Lipschitz continuous, in a similar way to that of proofs of (3.6) and (3.7), we obtain

$$
\left\|v_{n, 1}-u^{*}-\left(h\left(v_{n, 1}\right)-h\left(u^{*}\right)\right)\right\| \leq \sqrt{1-2 \varrho+\nu^{2}}\left\|v_{n, 1}-u^{*}\right\|
$$

and

(4.7) $\left\|g\left(v_{n, 1}\right)-g\left(u^{*}\right)-\rho\left(T\left(v_{n, 1}\right)-T\left(u^{*}\right)\right)\right\| \leq \sqrt{(1-2 \rho \delta) \tau^{2}+\rho^{2} \sigma^{2}}\left\|v_{n, 1}-u^{*}\right\|$.

By combining (4.5)-(4.7), we get

$$
\begin{aligned}
\left\|u_{n+1}-u^{*}\right\| \leq & \left(1-\alpha_{n, 1}-\beta_{n, 1}\right)\left\|u_{n}-u^{*}\right\|+\alpha_{n, 1} L \omega\left\|v_{n, 1}-u^{*}\right\| \\
& +\alpha_{n, 1} L b_{n}+\alpha_{n, 1}\left\|e_{n, 1}^{\prime}\right\|+\left\|e_{n, 1}^{\prime \prime}\right\|+\left\|r_{n, 1}\right\|+\beta_{n, 1} \Omega .
\end{aligned}
$$

Similarly, for each $i=1,2, \ldots, p-2$, we have

$$
\begin{aligned}
& \left\|v_{n, i}-u^{*}\right\| \\
\leq & \left(1-\alpha_{n, i+1}-\beta_{n, i+1}\right)\left\|u_{n}-u^{*}\right\|+\alpha_{n, i+1} L \omega\left\|v_{n, i+1}-u^{*}\right\| \\
& +\alpha_{n, i+1} L b_{n}+\alpha_{n, i+1}\left\|e_{n, i+1}^{\prime}\right\|+\left\|e_{n, i+1}^{\prime \prime}\right\|+\left\|r_{n, i+1}\right\|+\beta_{n, i+1} \Omega
\end{aligned}
$$


and

$$
\begin{aligned}
\left\|v_{n, p-1}-u^{*}\right\| \leq & \left(1-\alpha_{n, p}-\beta_{n, p}\right)\left\|u_{n}-u^{*}\right\|+\alpha_{n, p} L \omega\left\|u_{n}-u^{*}\right\| \\
& +\alpha_{n, p} L b_{n}+\alpha_{n, p}\left\|e_{n, p}^{\prime}\right\|+\left\|e_{n, p}^{\prime \prime}\right\|+\left\|r_{n, p}\right\|+\beta_{n, p} \Omega .
\end{aligned}
$$

From (4.9) and (4.10), we obtain

$$
\begin{aligned}
& \left\|v_{n, p-2}-u^{*}\right\| \\
\leq & \left(1-\alpha_{n, p-1}-\beta_{n, p-1}\right)\left\|u_{n}-u^{*}\right\|+\alpha_{n, p-1} L \omega\left\|v_{n, p-1}-u^{*}\right\| \\
& +\alpha_{n, p-1} L b_{n}+\alpha_{n, p-1}\left\|e_{n, p-1}^{\prime}\right\|+\left\|e_{n, p-1}^{\prime \prime}\right\|+\left\|r_{n, p-1}\right\|+\beta_{n, p-1} \Omega \\
\leq & \left(1-\alpha_{n, p-1}-\beta_{n, p-1}\right)\left\|u_{n}-u^{*}\right\|+\alpha_{n, p-1} L \omega\left(\left(1-\alpha_{n, p}-\beta_{n, p}\right)\left\|u_{n}-u^{*}\right\|\right. \\
& \left.+\alpha_{n, p} L \omega\left\|u_{n}-u^{*}\right\|+\alpha_{n, p} L b_{n}+\alpha_{n, p}\left\|e_{n, p}^{\prime}\right\|+\left\|e_{n, p}^{\prime \prime}\right\|+\left\|r_{n, p}\right\|+\beta_{n, p} \Omega\right) \\
& +\alpha_{n, p-1} L b_{n}+\alpha_{n, p-1}\left\|e_{n, p-1}^{\prime}\right\|+\left\|e_{n, p-1}^{\prime \prime}\right\|+\left\|r_{n, p-1}\right\|+\beta_{n, p-1} \Omega \\
= & \left(1-\alpha_{n, p-1}-\beta_{n, p-1}+\alpha_{n, p-1}\left(1-\alpha_{n, p}-\beta_{n, p}\right) L \omega+\alpha_{n, p-1} \alpha_{n, p} L^{2} \omega^{2}\right) \\
& \left\|u_{n}-u^{*}\right\|+\left(\alpha_{n, p-1} L+\alpha_{n, p-1} \alpha_{n, p} L^{2} \omega\right) b_{n}+\alpha_{n, p-1}\left\|e_{n, p-1}^{\prime}\right\| \\
& +\alpha_{n, p-1} \alpha_{n, p} L \omega\left\|e_{n, p}^{\prime}\right\|+\left\|e_{n, p-1}^{\prime \prime}\right\|+\alpha_{n, p-1} L \omega\left\|e_{n, p}^{\prime \prime}\right\|+\left\|r_{n, p-1}\right\| \\
& +\alpha_{n, p-1} L \omega\left\|r_{n, p}\right\|+\left(\beta_{n, p-1}+\alpha_{n, p-1} \beta_{n, p} L \omega\right) \Omega .
\end{aligned}
$$

In a similar way to that of proof of (4.11), by using (4.9) and (4.11), we get

$$
\begin{aligned}
& \left\|v_{n, p-3}-u^{*}\right\| \\
\leq & \left(1-\alpha_{n, p-2}-\beta_{n, p-2}+\alpha_{n, p-2}\left(1-\alpha_{n, p-1}-\beta_{n, p-1}\right) L \omega\right. \\
& \left.+\alpha_{n, p-2} \alpha_{n, p-1}\left(1-\alpha_{n, p}-\beta_{n, p}\right) L^{2} \omega^{2}+\alpha_{n, p-2} \alpha_{n, p-1} \alpha_{n, p} L^{3} \omega^{3}\right)\left\|u_{n}-u^{*}\right\| \\
& +\alpha_{n, p-2}\left\|e_{n, p-2}^{\prime}\right\|+\alpha_{n, p-2} \alpha_{n, p-1} L \omega\left\|e_{n, p-1}^{\prime}\right\| \\
& +\alpha_{n, p-2} \alpha_{n, p-1} \alpha_{n, p} L^{2} \omega^{2}\left\|e_{n, p}^{\prime}\right\|+\left(\alpha_{n, p-2} L+\alpha_{n, p-2} \alpha_{n, p-1} L^{2} \omega\right. \\
& \left.+\alpha_{n, p-2} \alpha_{n, p-1} \alpha_{n, p} L^{3} \omega^{2}\right) b_{n}+\left\|e_{n, p-2}^{\prime \prime}\right\|+\alpha_{n, p-2} L \omega\left\|e_{n, p-1}^{\prime \prime}\right\| \\
& +\alpha_{n, p-2} \alpha_{n, p-1} L^{2} \omega^{2}\left\|e_{n, p}^{\prime \prime}\right\|+\left\|r_{n, p-2}\right\|+\alpha_{n, p-2} L \omega\left\|r_{n, p-1}\right\| \\
& +\alpha_{n, p-2} \alpha_{n, p-1} L^{2} \omega^{2}\left\|r_{n, p}\right\|+\left(\beta_{n, p-2}+\alpha_{n, p-2} \beta_{n, p-1} L \omega\right. \\
& \left.+\alpha_{n, p-2} \alpha_{n, p-1} \beta_{n, p} L^{2} \omega^{2}\right) \Omega .
\end{aligned}
$$


Continuing in this way, we obtain

$$
\begin{aligned}
& \left\|v_{n, 1}-u^{*}\right\| \\
\leq & \left(1-\alpha_{n, 2}-\beta_{n, 2}+\alpha_{n, 2}\left(1-\alpha_{n, 3}-\beta_{n, 3}\right) L \omega\right. \\
& +\alpha_{n, 2} \alpha_{n, 3}\left(1-\alpha_{n, 4}-\beta_{n, 4}\right) L^{2} \omega^{2} \\
& +\cdots+\prod_{i=2}^{p-1} \alpha_{n, i}\left(1-\alpha_{n, p}-\beta_{n, p}\right) L^{p-2} \omega^{p-2} \\
& \left.+\prod_{i=2}^{p} \alpha_{n, i} L^{p-1} \omega^{p-1}\right)\left\|u_{n}-u^{*}\right\| \\
& +\left(\alpha_{n, 2} L+\alpha_{n, 2} \alpha_{n, 3} L^{2} \omega+\alpha_{n, 2} \alpha_{n, 3} \alpha_{n, 4} L^{3} \omega^{2}\right. \\
& \left.+\cdots+\prod_{i=2}^{p} \alpha_{n, i} L^{p-1} \omega^{p-2}\right) b_{n} \\
& +\alpha_{n, 2}\left\|e_{n, 2}^{\prime}\right\|+\alpha_{n, 2} \alpha_{n, 3} L \omega\left\|e_{n, 3}^{\prime}\right\|+\cdots+\prod_{i=2}^{p} \alpha_{n, i} L^{p-2} \omega^{p-2}\left\|e_{n, p}^{\prime}\right\| \\
& +\left\|e_{n, 2}^{\prime \prime}\right\|+\alpha_{n, 2} L \omega\left\|e_{n, 3}^{\prime \prime}\right\|+\alpha_{n, 2} \alpha_{n, 3} L^{2} \omega^{2}\left\|e_{n, 4}^{\prime \prime}\right\| \\
& +\cdots+\prod_{i=2}^{p-1} \alpha_{n, i} L^{p-2} \omega^{p-2}\left\|e_{n, p}^{\prime \prime}\right\| \\
& +\left\|r_{n, 2}\right\|+\alpha_{n, 2} L \omega\left\|r_{n, 3}\right\|+\alpha_{n, 2} \alpha_{n, 3} L^{2} \omega^{2}\left\|r_{n, 4}\right\| \\
& +\cdots+\prod_{i=2}^{p-1} \alpha_{n, i} L^{p-2} \omega^{p-2}\left\|r_{n, p}\right\| \\
& +\left(\beta_{n, 2}+\alpha_{n, 2} \beta_{n, 3} L \omega+\alpha_{n, 2} \alpha_{n, 3} \beta_{n, 4} L^{2} \omega^{2}\right. \\
& \left.+\cdots+\prod_{i=2}^{p-1} \alpha_{n, i} \beta_{n, p} L^{p-2} \omega^{p-2}\right) \Omega .
\end{aligned}
$$

By (4.8) and (4.13), we deduce

$$
\begin{aligned}
&\left\|u_{n+1}-u^{*}\right\| \\
& \leq\left(1-\alpha_{n, 1}-\beta_{n, 1}\right)\left\|u_{n}-u^{*}\right\|+\alpha_{n, 1} L \omega\left\|v_{n, 1}-u^{*}\right\| \\
&+\alpha_{n, 1} L b_{n}+\alpha_{n, 1}\left\|e_{n, 1}^{\prime}\right\|+\left\|e_{n, 1}^{\prime \prime}\right\|+\left\|r_{n, 1}\right\|+\beta_{n, 1} \Omega \\
& \leq\left(1-\alpha_{n, 1}-\beta_{n, 1}+\alpha_{n, 1}\left(1-\alpha_{n, 2}-\beta_{n, 2}\right) L \omega\right. \\
&+\alpha_{n, 1} \alpha_{n, 2}\left(1-\alpha_{n, 3}-\beta_{n, 3}\right) L^{2} \omega^{2} \\
&\left.+\ldots+\prod_{i=1}^{p-1} \alpha_{n, i}\left(1-\alpha_{n, p}-\beta_{n, p}\right) L^{p-1} \omega^{p-1}+\prod_{i=1}^{p} \alpha_{n, i} L^{p} \omega^{p}\right)\left\|u_{n}-u^{*}\right\| \\
&+\left(\alpha_{n, 1} L+\alpha_{n, 1} \alpha_{n, 2} L^{2} \omega+\alpha_{n, 1} \alpha_{n, 2} \alpha_{n, 3} L^{3} \omega^{2}+\ldots+\prod_{i=1}^{p} \alpha_{n, i} L^{p} \omega^{p-1}\right) b_{n} \\
&+\alpha_{n, 1}\left\|e_{n, 1}^{\prime}\right\|+\alpha_{n, 1} \alpha_{n, 2} L \omega\left\|e_{n, 2}^{\prime}\right\|+\cdots+\prod_{i=1}^{p} \alpha_{n, i} L^{p-1} \omega^{p-1}\left\|e_{n, p}^{\prime}\right\| \\
&+\left\|e_{n, 1}^{\prime \prime}\right\|+\alpha_{n, 1} L \omega\left\|e_{n, 2}^{\prime \prime}\right\|+\alpha_{n, 1} \alpha_{n, 2} L^{2} \omega^{2}\left\|e_{n, 3}^{\prime \prime}\right\|
\end{aligned}
$$




$$
\begin{aligned}
& +\cdots+\prod_{i=1}^{p-1} \alpha_{n, i} L^{p-1} \omega^{p-1}\left\|e_{n, p}^{\prime \prime}\right\| \\
& +\left\|r_{n, 1}\right\|+\alpha_{n, 1} L \omega\left\|r_{n, 2}\right\|+\alpha_{n, 1} \alpha_{n, 2} L^{2} \omega^{2}\left\|r_{n, 3}\right\|+\ldots+\prod_{i=1}^{p-1} \alpha_{n, i} L^{p-1} \omega^{p-1}\left\|r_{n, p}\right\| \\
& +\left(\beta_{n, 1}+\alpha_{n, 1} \beta_{n, 2} L \omega+\alpha_{n, 1} \alpha_{n, 2} \beta_{n, 3} L^{2} \omega^{2}+\cdots+\prod_{i=1}^{p-1} \alpha_{n, i} \beta_{n, p} L^{p-1} \omega^{p-1}\right) \Omega \\
& \leq \\
& \left.+1-(1-L \omega) \prod_{i=1}^{p} \alpha_{n, i} L^{p-1} \omega^{p-1}\right]\left\|u_{n}-u^{*}\right\|+\sum_{i=1}^{p} \prod_{j=1}^{i} \alpha_{n, j} L^{i} \omega^{i-1} b_{n} \\
& +\sum_{i=1}^{p} \prod_{j=1}^{i} \alpha_{n, j} L^{i-1} \omega^{i-1}\left\|e_{n, i}^{\prime}\right\|+\left\|e_{n, 1}^{\prime \prime}\right\|+\sum_{i=2}^{p} \prod_{j=1}^{i-1} \alpha_{n, j} L^{i-1} \omega^{i-1}\left\|e_{n, i}^{\prime \prime}\right\|+\left\|r_{n, 1}\right\| \\
& +\sum_{i=2}^{p} \prod_{j=1}^{i-1} \alpha_{n, j} L^{i-1} \omega^{i-1}\left\|r_{n, i}\right\|+\left(\beta_{n, 1}+\sum_{i=2}^{p} \prod_{j=1}^{i-1} \alpha_{n, j} \beta_{n, i} L^{i-1} \omega^{i-1}\right) \Omega \\
& \leq \\
& {\left[1-(1-L \omega) \prod_{i=1}^{p} \alpha_{n, i} L^{p-1} \omega^{p-1}\right]\left\|u_{n}-u^{*}\right\|} \\
& +(1-L \omega) \prod_{i=1}^{p} \alpha_{n, i} L^{p-1} \omega^{p-1} \frac{\sum_{i=1}^{p} \prod_{j=1}^{i} \alpha_{n, j} L^{i} \omega^{i-1} b_{n}+\sum_{i=1}^{p} \prod_{j=1}^{i} \alpha_{n, j} L^{i-1} \omega^{i-1}\left\|e_{n, i}^{\prime}\right\|}{\alpha(1-L \omega) L^{p-1} \omega^{p-1}} \\
& +\sum_{i=2}^{p} \prod_{j=1}^{i-1} \alpha_{n, j} L^{i-1} \omega^{i-1}\left\|e_{n, i}^{\prime \prime}\right\|+\sum_{i=2}^{p} \prod_{j=1}^{i-1} \alpha_{n, j} L^{i-1} \omega^{i-1}\left\|r_{n, i}\right\| \\
& +\left\|e_{n, 1}^{\prime \prime}\right\|+\left\|r_{n, 1}\right\|+\left(\beta_{n, 1}+\sum_{i=2}^{p} \prod_{j=1}^{i-1} \alpha_{n, j} \beta_{n, i} L^{i-1} \omega^{i-1}\right) \Omega .
\end{aligned}
$$

Since $L \omega<1$ and $\lim _{n \rightarrow \infty} b_{n}=0$, in view of (4.3), it is evident that all the conditions of Lemma 4.7 are satisfied, and so, Lemma 4.7 and (4.14) guarantee that $u_{n} \rightarrow u^{*}$ as $n \rightarrow \infty$. Accordingly, the sequence $\left\{u_{n}\right\}_{n=0}^{\infty}$ generated by Algorithm 4.1 converges strongly to a unique solution $u^{*}$ of problem (2.1), that is, the only element $u^{*}$ of $\operatorname{Fix}(S) \cap \operatorname{EGNQVI}(\mathcal{H}, T, g, h)$.

Theorem 4.9. Let $T, g, h$ and $\rho$ be the same as in Theorem 3.2 and let all the conditions of Theorem 3.2 hold. Then the iterative sequence $\left\{u_{n}\right\}_{n=0}^{\infty}$ generated by Algorithm 4.3 converges strongly to a unique solution of problem (2.1).

Remark 4.10. Theorems 4.8 and 4.9 generalize and improve Theorem 4.1 in [14].

\section{Extended General Wiener-hopf Equations and Iterative Methods}

In this section, we introduce a new class of extended general Wiener-Hopf equations and establish the equivalence between the aforesaid class and the class of the extended general nonlinear quasi-variational inequalities. By using this equivalence, we suggest and analyze some new perturbed projection iterative algorithms for solving problem (2.1). 
Let $T, g, h$ and $\rho$ be the same as in problem (2.1) and suppose that the inverse of the operator $h$ exists. Associated with problem (2.1), for a given $u \in \mathcal{H}$ with $h(u) \in K(u)$, we consider the problem of finding $z \in \mathcal{H}$ such that

$$
T h^{-1} P_{K(u)} z+\rho^{-1} Q_{K(u)} z=0,
$$

where $Q_{K(u)}=I-g h^{-1} P_{K(u)}$ and $I$ is the identity operator.

Problem (5.1) is called extended general Wiener-Hopf equation (EGWHE) associated with (2.1). We denote by $\operatorname{EGWHE}(\mathcal{H}, T, g, h)$ the set of the solutions of the extended general Wiener-Hopf equation (5.1).

Some special cases of the problem (5.1) can be found in $[14,20]$ and the references therein.

Remark 5.1. It has been shown that the Wiener-Hopf equations have played an important and significant role in developing several numerical techniques for solving variational inequalities and related optimizations problems, see, for example, $[9,14$, $18,20]$ and the references therein.

We establish the equivalence between the extended general nonlinear quasi-variational inequality (2.1) and the extended general Wiener-Hopf equation (5.1).

Lemma 5.2. Let $T, g, h$ and $\rho$ be the same as in problem (2.1) and suppose that the inverse of the operator $h$ exists. Then $u \in \mathcal{H}$ with $h(u) \in K(u)$ is a solution of problem (2.1) if and only if the extended general Wiener-Hopf equation (5.1) has a solution $z \in \mathcal{H}$ satisfying

$$
h(u)=P_{K(u)} z, \quad z=g(u)-\rho T(u) .
$$

Proof. Let $u \in \mathcal{H}$ with $h(u) \in K(u)$ be a solution of problem (2.1). By using Lemma 3.1, we obtain

$$
h(u)=P_{K(u)}(g(u)-\rho T(u)) .
$$

Taking $z=g(u)-\rho T(u)$ in (5.2), we have $h(u)=P_{K(u)} z$, which leads to

$$
u=h^{-1} P_{K(u)} z .
$$

From (5.3) and this fact that $z=g(u)-\rho T(u)$, it follows that

$$
z=g h^{-1} P_{K(u)} z-\rho T h^{-1} P_{K(u)} z .
$$

Evidently, the above equality is equivalent to

$$
T h^{-1} P_{K(u)} z+\rho^{-1} Q_{K(u)} z=0,
$$


where $Q_{K(u)}$ is the same as in (5.1). Now, (5.4) guarantees that $z \in \mathcal{H}$ is a solution of the extended general Wiener-Hopf equation (5.1).

Conversely, if $z \in \mathcal{H}$ is a solution of problem (5.1) satisfying

$$
h(u)=P_{K(u)} z, \quad z=g(u)-\rho T(u),
$$

then it follows from Lemma 3.1 that $u \in \mathcal{H}$ with $h(u) \in K(u)$ is a solution of problem (2.1).

By using problem (5.1) and Lemma 5.2, we obtain some fixed point formulations for constructing a number of new perturbed projection iterative algorithms for solving problem (2.1).

(I) By using (5.1) and Lemma 5.2, we have

$$
\begin{aligned}
T h^{-1} P_{K(u)} z+\rho^{-1} Q_{K(u)} z=0 & \Leftrightarrow \rho T h^{-1} P_{K(u)} z+Q_{K(u)} z=0 \\
& \Leftrightarrow \rho T h^{-1} P_{K(u)} z+z-g h^{-1} P_{K(u)} z=0 \\
& \Leftrightarrow z=g h^{-1} P_{K(u)} z-\rho T h^{-1} P_{K(u)} z \\
& \Leftrightarrow z=g(u)-\rho T(u) .
\end{aligned}
$$

By using this fixed point formulation, we define the following finite step projection iterative algorithm with mixed errors for solving problem (2.1).

Algorithm 5.3. Let $T, g, h$ and $\rho$ be the same as in problem (2.1) such that $h$ is an onto mapping. For an arbitrary chosen initial point $z_{0} \in \mathcal{H}$, compute the iterative sequence $\left\{z_{n}\right\}_{n=0}^{\infty}$ in the following way:

$$
\left\{\begin{aligned}
& h\left(u_{n}\right)= S^{n} P_{K\left(u_{n}\right)} z_{n}, \\
& z_{n+1}=\left(1-\alpha_{n, 1}-\beta_{n, 1}\right) z_{n}+\alpha_{n, 1}\left(g\left(v_{n, 1}\right)\right. \\
&\left.-\rho T\left(v_{n, 1}\right)+e_{n, 1}\right)+\beta_{n, 1} l_{n, 1}+r_{n, 1}, \\
& v_{n, i}=\left(1-\alpha_{n, i+1}-\beta_{n, i+1}\right) z_{n}+\alpha_{n, i+1}\left(g\left(v_{n, i+1}\right)\right. \\
&\left.-\rho T\left(v_{n, i+1}\right)+e_{n, i+1}\right)+\beta_{n, i+1} l_{n, i+1}+r_{n, i+1}, \\
& \vdots \quad\left(1-\alpha_{n, p}-\beta_{n, p}\right) z_{n}+\alpha_{n, p}\left(g\left(u_{n}\right)\right. \\
& v_{n, p-1}=\left.-\rho T\left(u_{n}\right)+e_{n, p}\right)+\beta_{n, p} l_{n, p}+r_{n, p}, \\
& i=1,2, \ldots, p-2,
\end{aligned}\right.
$$

where $S,\left\{\alpha_{n, i}\right\}_{n=0}^{\infty},\left\{\beta_{n, i}\right\}_{n=0}^{\infty},\left\{e_{n, i}\right\}_{n=0}^{\infty},\left\{l_{n, i}\right\}_{n=0}^{\infty},\left\{r_{n, i}\right\}_{n=0}^{\infty}(i=1,2, \ldots p)$ are the same as in Algorithm 4.1. 
(II) It follows from (5.1) and Lemma 5.2 that

$$
\begin{aligned}
& T h^{-1} P_{K(u)} z+\rho^{-1} Q_{K(u)} z=0 \\
\Leftrightarrow & Q_{K(u)} z=Q_{K(u)} z-T h^{-1} P_{K(u)} z-\rho^{-1} Q_{K(u)} z \\
\Leftrightarrow & Q_{K(u)} z=-T h^{-1} P_{K(u)} z+\left(1-\rho^{-1}\right) Q_{K(u)} z \\
\Leftrightarrow & z=g h^{-1} P_{K(u)} z-T h^{-1} P_{K(u)} z+\left(1-\rho^{-1}\right) Q_{K(u)} z \\
\Leftrightarrow & z=g(u)-T(u)+\left(1-\rho^{-1}\right) Q_{K(u)} z .
\end{aligned}
$$

This fixed point formulation enables us to construct the following $p$-step projection iterative algorithm with mixed errors for solving problem (2.1).

Algorithm 5.4. Let $T, g, h$ and $\rho$ be the same as in Algorithm 5.3. For an arbitrary chosen initial point $z_{0} \in \mathcal{H}$, compute the iterative sequence $\left\{z_{n}\right\}_{n=0}^{\infty}$ in the following way:

$$
\left\{\begin{array}{l}
h\left(u_{n}\right)=S^{n} P_{K\left(u_{n}\right)} z_{n}, \\
z_{n+1}=\left(1-\alpha_{n, 1}-\beta_{n, 1}\right) z_{n}+\alpha_{n, 1}\left(\Phi\left(v_{n, 1}, z_{n}\right)+e_{n, 1}\right)+\beta_{n, 1} l_{n, 1}+r_{n, 1}, \\
v_{n, i}=\left(1-\alpha_{n, i+1}-\beta_{n, i+1}\right) z_{n}+\alpha_{n, i+1}\left(\Phi\left(v_{n, i+1}, z_{n}\right)+e_{n, i+1}\right) \\
\quad \quad+\beta_{n, i+1} l_{n, i+1}+r_{n, i+1}, \\
\quad \vdots \quad \\
v_{n, p-1}=\left(1-\alpha_{n, p}-\beta_{n, p}\right) z_{n}+\alpha_{n, p}\left(\Phi\left(u_{n}, z_{n}\right)+e_{n, p}\right)+\beta_{n, p} l_{n, p}+r_{n, p}, \\
i=1,2, \ldots, p-2,
\end{array}\right.
$$

where

$$
\left\{\begin{array}{l}
\Phi\left(v_{n, i}, z_{n}\right)=g\left(v_{n, i}\right)-T\left(v_{n, i}\right)+\left(1-\rho^{-1}\right) Q_{K\left(v_{n, i}\right)} z_{n} \\
\Phi\left(u_{n}, z_{n}\right)=g\left(u_{n}\right)-T\left(u_{n}\right)+\left(1-\rho^{-1}\right) Q_{K\left(u_{n}\right)} z_{n} \\
i=1,2, \ldots, p-1
\end{array}\right.
$$

and $S,\left\{\alpha_{n, i}\right\}_{n=0}^{\infty},\left\{\beta_{n, i}\right\}_{n=0}^{\infty},\left\{e_{n, i}\right\}_{n=0}^{\infty},\left\{l_{n, i}\right\}_{n=0}^{\infty},\left\{r_{n, i}\right\}_{n=0}^{\infty}(i=1,2, \ldots p)$ are the same as in Algorithm 4.2.

Algorithm 5.5. Let $T, g, h$ and $\rho$ be the same as in Algorithm 5.3. For an arbitrary chosen initial point $z_{0} \in \mathcal{H}$, compute the iterative sequence $\left\{z_{n}\right\}_{n=0}^{\infty}$ in the following way:

$$
\left\{\begin{array}{l}
h\left(u_{n}\right)=S^{n} P_{K\left(u_{n}\right)} z_{n}, \\
z_{n+1}=\left(1-\alpha_{n}\right) z_{n}+\alpha_{n}\left(g\left(u_{n}\right)-\rho T\left(u_{n}\right)\right),
\end{array}\right.
$$

where $S$ and $\left\{\alpha_{n}\right\}_{n=0}^{\infty}$ are the same as in Algorithm 4.2. 
Algorithm 5.6. Let $T, g, h$ and $\rho$ be the same as in Algorithm 5.3. For an arbitrary chosen initial point $z_{0} \in \mathcal{H}$, compute the iterative sequence $\left\{z_{n}\right\}_{n=0}^{\infty}$ in the following way:

$$
\left\{\begin{array}{l}
h\left(u_{n}\right)=S^{n} P_{K\left(u_{n}\right)} z_{n} \\
z_{n+1}=\left(1-\alpha_{n}\right) z_{n}+\alpha_{n}\left(g\left(u_{n}\right)-T\left(u_{n}\right)+\left(1-\rho^{-1}\right) Q_{K\left(u_{n}\right)} z_{n}\right),
\end{array}\right.
$$

where $S$ and $\left\{\alpha_{n}\right\}_{n=0}^{\infty}$ are the same as in Algorithm 4.2.

(III) Let the operators $T$ and $h$ be linear and suppose that the inverses of $T$ and $g$, that is, $T^{-1}$ and $g^{-1}$ exist. Then (5.1) may be written as:

$$
\begin{aligned}
T h^{-1} P_{K(u)} z+\rho^{-1} Q_{K(u)} z=0 & \Leftrightarrow T\left(g^{-1}\left(z-Q_{K(u)} z\right)\right)+\rho^{-1} Q_{K(u)} z=0 \\
& \Leftrightarrow g^{-1}\left(z-Q_{K(u)} z\right)=T^{-1}\left(-\rho^{-1} Q_{K(u)} z\right) \\
& \Leftrightarrow z-Q_{K(u)} z=g\left(-\rho^{-1} T^{-1} Q_{K(u)} z\right) \\
& \Leftrightarrow z=Q_{K(u)} z-\rho^{-1} g T^{-1} Q_{K(u)} z \\
& \Leftrightarrow z=\left(I-\rho^{-1} g T^{-1}\right) Q_{K(u)} z .
\end{aligned}
$$

This fixed point formulation allows us to construct the following projection iterative algorithm for solving problem (2.1).

Algorithm 5.7. Assume that $T, g, h$ and $\rho$ are the same as in Algorithm 5.3. For any $z_{0} \in \mathcal{H}$, define the iterative sequence $\left\{z_{n}\right\}_{n=0}^{\infty}$ by the following iterative process:

$$
z_{n+1}=\left(1-\alpha_{n}\right) z_{n}+\alpha_{n}\left(I-\rho^{-1} g T^{-1}\right) Q_{K\left(u_{n}\right)} z_{n},
$$

where $S$ and $\left\{\alpha_{n}\right\}_{n=0}^{\infty}$ are the same as in Algorithm 4.2.

Remark 5.8. Algorithms 5.1-5.6 in [14] are special cases of Algorithms 5.3-5.7. Similar to Remark 4.5, for a suitable and appropriate choice of the sequences $\left\{e_{n, i}\right\}_{n=0}^{\infty}$, $\left\{l_{n, i}\right\}_{n=0}^{\infty}$ and $\left\{r_{n, i}\right\}_{n=0}^{\infty}(i=1,2 \ldots, p)$, Algorithms 5.3 and 5.4 reduce to algorithms with mean errors and without errors.

\section{A Strongly Convergence Theorem}

In the present section, we discuss the convergence analysis of iterative sequence generated by the perturbed projection iterative Algorithm 5.3. In a similar way, one can study the convergence analysis of iterative sequences generated by Algorithms 5.4-5.7.

Theorem 6.1. Let $T, g, h$ and $\rho$ be the same as in problem (2.1) and assume that all the conditions of Theorem 3.2 hold. Let $S: \mathcal{H} \rightarrow \mathcal{H}$ be a nearly uniformly L-Lipschitzian mapping with the sequence $\left\{b_{n}\right\}_{n=0}^{\infty}$ such that for each $u \in \operatorname{EGNQVI}(\mathcal{H}, T, g, h), h(u) \in \operatorname{Fix}(S)$. Further, assume that $L \omega<1$, where 
$\omega$ is the same as in (3.9). If there exists a constant $\alpha>0$ such that $\prod_{i=1}^{p} \alpha_{n, i} \geq \alpha$, for each $n \geq 0$, then the iterative sequence $\left\{z_{n}\right\}_{n=0}^{\infty}$ generated by Algorithm 5.3 converges strongly to an element $z \in \operatorname{EGWHE}(\mathcal{H}, T, g, h)$.

Proof. According to Theorem 3.2, Problem (2.1) has a unique solution $u^{*} \in \mathcal{H}$ with $h\left(u^{*}\right) \in K\left(u^{*}\right)$. Hence, in view of Lemma 5.2, there exists a unique point $z \in \mathcal{H}$ satisfying

$$
h\left(u^{*}\right)=P_{K\left(u^{*}\right)} z, \quad z=g\left(u^{*}\right)-\rho T\left(u^{*}\right) .
$$

Since $h\left(u^{*}\right) \in \operatorname{Fix}(S)$, it follows from (6.1) that for each $n \geq 0$

$$
h\left(u^{*}\right)=S^{n} P_{K\left(u^{*}\right)} z, \quad z=g\left(u^{*}\right)-\rho T\left(u^{*}\right) .
$$

Let $\Omega=\sup _{n \geq 0}\left\{\left\|l_{n, i}-z\right\|,\left\|z-u^{*}\right\|: i=1,2, \ldots, p\right\}$. It follows from (5.5), (6.1) and the assumptions that

$$
\begin{aligned}
& \left\|z_{n+1}-z\right\| \\
\leq & \left(1-\alpha_{n, 1}-\beta_{n, 1}\right)\left\|z_{n}-z\right\|+\alpha_{n, 1}\left\|g\left(v_{n, 1}\right)-g\left(u^{*}\right)-\rho\left(T\left(v_{n, 1}\right)-T\left(u^{*}\right)\right)\right\| \\
+ & \beta_{n, 1}\left\|l_{n, 1}-z\right\|+\alpha_{n, 1}\left\|e_{n, 1}\right\|+\left\|r_{n, 1}\right\| \\
\leq & \left(1-\alpha_{n, 1}-\beta_{n, 1}\right)\left\|z_{n}-z\right\|+\alpha_{n, 1} \sqrt{(1-2 \rho \delta) \tau^{2}+\rho^{2} \sigma^{2}}\left\|v_{n, 1}-u^{*}\right\| \\
& +\beta_{n, 1}\left\|l_{n, 1}-z\right\|+\alpha_{n, 1}\left(\left\|e_{n, 1}^{\prime}\right\|+\left\|e_{n, 1}^{\prime \prime}\right\|\right)+\left\|r_{n, 1}\right\| \\
\leq & \left(1-\alpha_{n, 1}-\beta_{n, 1}\right)\left\|z_{n}-z\right\|+\alpha_{n, 1} \sqrt{(1-2 \rho \delta) \tau^{2}+\rho^{2} \sigma^{2}}\left\|v_{n, 1}-z\right\| \\
& +\alpha_{n, 1} \sqrt{(1-2 \rho \delta) \tau^{2}+\rho^{2} \sigma^{2}}\left\|z-u^{*}\right\|+\alpha_{n, 1}\left\|e_{n, 1}^{\prime}\right\|+\left\|e_{n, 1}^{\prime \prime}\right\|+\left\|r_{n, 1}\right\|+\beta_{n, 1} \Omega \\
\leq & \left(1-\alpha_{n, 1}-\beta_{n, 1}\right)\left\|z_{n}-z\right\|+\alpha_{n, 1} \sqrt{(1-2 \rho \delta) \tau^{2}+\rho^{2} \sigma^{2}}\left\|v_{n, 1}-z\right\| \\
& +\left(\alpha_{n, 1} \sqrt{(1-2 \rho \delta) \tau^{2}+\rho^{2} \sigma^{2}}+\beta_{n, 1}\right) \Omega+\alpha_{n, 1}\left\|e_{n, 1}^{\prime}\right\|+\left\|e_{n, 1}^{\prime \prime}\right\|+\left\|r_{n, 1}\right\| .
\end{aligned}
$$

Similarly, for each $i=1,2, \ldots, p-2$, we have

$$
\begin{aligned}
\left\|v_{n, i}-z\right\| \leq & \left(1-\alpha_{n, i+1}-\beta_{n, i+1}\right)\left\|z_{n}-z\right\| \\
& +\alpha_{n, i+1} \sqrt{(1-2 \rho \delta) \tau^{2}+\rho^{2} \sigma^{2}}\left\|v_{n, i+1}-z\right\| \\
& +\left(\alpha_{n, i+1} \sqrt{(1-2 \rho \delta) \tau^{2}+\rho^{2} \sigma^{2}}+\beta_{n, i+1}\right) \Omega \\
& +\alpha_{n, i+1}\left\|e_{n, i+1}^{\prime}\right\|+\left\|e_{n, i+1}^{\prime \prime}\right\|+\left\|r_{n, i+1}\right\|
\end{aligned}
$$

and$$
\left\|v_{n, p-1}-z\right\|
$$$$
\text { (6.5) } \leq\left(1-\alpha_{n, p}-\beta_{n, p}\right)\left\|z_{n}-z\right\|+\alpha_{n, p} \sqrt{(1-2 \rho \delta) \tau^{2}+\rho^{2} \sigma^{2}}\left\|u_{n}-u^{*}\right\|
$$$$
+\alpha_{n, p}\left\|e_{n, p}^{\prime}\right\|+\left\|e_{n, p}^{\prime \prime}\right\|+\left\|r_{n, p}\right\|+\beta_{n, p} \Omega \text {. }
$$ 
By using (5.5) and (6.2), we obtain an estimation for $\left\|u_{n}-u^{*}\right\|$ in the following way:

$$
\begin{aligned}
\left\|u_{n}-u^{*}\right\| \leq & \left\|u_{n}-u^{*}-\left(h\left(u_{n}\right)-h\left(u^{*}\right)\right)\right\|+\left\|S^{n} P_{K\left(u_{n}\right)} z_{n}-S^{n} P_{K\left(u^{*}\right)} z\right\| \\
\leq & \sqrt{1-2 \varrho+\nu^{2}}\left\|u_{n}-u^{*}\right\|+L\left(\left\|P_{K\left(u_{n}\right)} z_{n}-P_{K\left(u^{*}\right)} z\right\|+b_{n}\right) \\
\leq & \sqrt{1-2 \varrho+\nu^{2}}\left\|u_{n}-u^{*}\right\|+L\left(\left\|P_{K\left(u_{n}\right)} z-P_{K\left(u^{*}\right)} z\right\|\right. \\
& \left.+\left\|P_{K\left(u_{n}\right)} z_{n}-P_{K\left(u_{n}\right)} z\right\|+b_{n}\right) \\
\leq & \sqrt{1-2 \varrho+\nu^{2}}\left\|u_{n}-u^{*}\right\|+L\left(\varsigma\left\|u_{n}-u^{*}\right\|+\left\|z_{n}-z\right\|+b_{n}\right) \\
\leq & \left(L \varsigma+\sqrt{1-2 \varrho+\nu^{2}}\right)\left\|u_{n}-u^{*}\right\|+L\left(\left\|z_{n}-z\right\|+b_{n}\right),
\end{aligned}
$$

which leads to

$$
\left\|u_{n}-u^{*}\right\| \leq \frac{L}{1-L \varsigma-\sqrt{1-2 \varrho+\nu^{2}}}\left\|z_{n}-z\right\|+\frac{L b_{n}}{1-L \varsigma-\sqrt{1-2 \varrho+\nu^{2}}}
$$

By (6.5) and (6.6), we have

$$
\begin{aligned}
\left\|v_{n, p-1}-z\right\| \leq & \left(1-\alpha_{n, p}-\beta_{n, p}\right)\left\|z_{n}-z\right\| \\
& +\alpha_{n, p} L \frac{\sqrt{(1-2 \rho \delta) \tau^{2}+\rho^{2} \sigma^{2}}}{1-L \varsigma-\sqrt{1-2 \varrho+\nu^{2}}}\left\|z_{n}-z\right\| \\
& +\alpha_{n, p} L \frac{\sqrt{(1-2 \rho \delta) \tau^{2}+\rho^{2} \sigma^{2}}}{1-L \varsigma-\sqrt{1-2 \varrho+\nu^{2}}} b_{n} \\
& +\alpha_{n, p}\left\|e_{n, p}^{\prime}\right\|+\left\|e_{n, p}^{\prime \prime}\right\|+\left\|r_{n, p}\right\|+\beta_{n, p} \Omega \\
= & \left(1-\alpha_{n, p}-\beta_{n, p}\right)\left\|z_{n}-z\right\|+\alpha_{n, p} L \vartheta\left\|z_{n}-z\right\| \\
& +\alpha_{n, p} L \vartheta b_{n}+\alpha_{n, p}\left\|e_{n, p}^{\prime}\right\|+\left\|e_{n, p}^{\prime \prime}\right\|+\left\|r_{n, p}\right\|+\beta_{n, p} \Omega,
\end{aligned}
$$

where $\vartheta=\frac{\sqrt{(1-2 \rho \delta) \tau^{2}+\rho^{2} \sigma^{2}}}{1-L \varsigma-\sqrt{1-2 \varrho+\nu^{2}}}$. From $L \omega<1$ and the condition (3.3), we deduce that $\vartheta<1$. By

$$
\omega=\varsigma+\sqrt{1-2 \varrho+\nu^{2}}+\sqrt{(1-2 \rho \delta) \tau^{2}+\rho^{2} \sigma^{2}}<1
$$

we have

$$
\gamma=\sqrt{(1-2 \rho \delta) \tau^{2}+\rho^{2} \sigma^{2}}<1 .
$$

Applying (6.8), the inequality (6.4), for each $i=1,2, \ldots, p-2$, can be written as follows:

$$
\begin{aligned}
& \left\|v_{n, i}-z\right\| \\
\leq & \left(1-\alpha_{n, i+1}-\beta_{n, i+1}\right)\left\|z_{n}-z\right\|+\alpha_{n, i+1} \gamma\left\|v_{n, i+1}-z\right\| \\
& +\alpha_{n, i+1}\left\|e_{n, i+1}^{\prime}\right\|+\left\|e_{n, i+1}^{\prime \prime}\right\|+\left\|r_{n, i+1}\right\|+\left(\alpha_{n, i+1} \gamma+\beta_{n, i+1}\right) \Omega .
\end{aligned}
$$


From (6.7) and (6.9), we obtain

$$
\begin{aligned}
\| & v_{n, p-2}-z \| \\
\leq & \left(1-\alpha_{n, p-1}-\beta_{n, p-1}\right)\left\|z_{n}-z\right\|+\alpha_{n, p-1} \gamma\left(\left(1-\alpha_{n, p}-\beta_{n, p}\right)\left\|z_{n}-z\right\|\right. \\
& \left.+\alpha_{n, p} L \vartheta\left\|z_{n}-z\right\|+\alpha_{n, p} L \vartheta b_{n}+\alpha_{n, p}\left\|e_{n, p}^{\prime}\right\|+\left\|e_{n, p}^{\prime \prime}\right\|+\left\|r_{n, p}\right\|+\beta_{n, p} \Omega\right) \\
& +\alpha_{n, p-1}\left\|e_{n, p-1}^{\prime}\right\|+\left\|e_{n, p-1}^{\prime \prime}\right\|+\left\|r_{n, p-1}\right\|+\left(\alpha_{n, p-1} \gamma+\beta_{n, p-1}\right) \Omega \\
= & \left(1-\alpha_{n, p-1}-\beta_{n, p-1}+\alpha_{n, p-1}\left(1-\alpha_{n, p}-\beta_{n, p}\right) \gamma+\alpha_{n, p-1} \alpha_{n, p} \gamma L \vartheta\right)\left\|z_{n}-z\right\| \\
& +\alpha_{n, p-1} \alpha_{n, p} \gamma L \vartheta b_{n}+\alpha_{n, p-1} \alpha_{n, p} \gamma\left\|e_{n, p}^{\prime}\right\|+\alpha_{n, p-1}\left\|e_{n, p-1}^{\prime}\right\| \\
& +\alpha_{n, p-1} \gamma\left\|e_{n, p}^{\prime \prime}\right\|+\left\|e_{n, p-1}^{\prime \prime}\right\|+\alpha_{n, p-1} \gamma\left\|r_{n, p}\right\|+\left\|r_{n, p-1}\right\| \\
& +\left(\alpha_{n, p-1} \beta_{n, p} \gamma+\alpha_{n, p-1} \gamma+\beta_{n, p-1}\right) \Omega .
\end{aligned}
$$

Similarly, by using (6.9) and (6.10), we get

$$
\begin{aligned}
& \quad\left\|v_{n, p-3}-z\right\| \\
& \leq\left(1-\alpha_{n, p-2}-\beta_{n, p-2}+\alpha_{n, p-2}\left(1-\alpha_{n, p-1}-\beta_{n, p-1}\right) \gamma\right. \\
& \left.\quad+\alpha_{n, p-2} \alpha_{n, p-1}\left(1-\alpha_{n, p}-\beta_{n, p}\right) \gamma^{2}+\alpha_{n, p-2} \alpha_{n, p-1} \alpha_{n, p} \gamma^{2} L \vartheta\right)\left\|z_{n}-z\right\| \\
& \quad+\alpha_{n, p-2} \alpha_{n, p-1} \alpha_{n, p} \gamma^{2}\left\|e_{n, p}^{\prime}\right\|+\alpha_{n, p-2} \alpha_{n, p-1} \gamma\left\|e_{n, p-1}^{\prime}\right\|+\alpha_{n, p-2}\left\|e_{n, p-2}^{\prime}\right\| \\
& \quad+\alpha_{n, p-2} \alpha_{n, p-1} \alpha_{n, p} \gamma^{2} L \vartheta b_{n}+\alpha_{n, p-2} \alpha_{n, p-1} \gamma^{2}\left\|e_{n, p}^{\prime \prime}\right\| \\
& \quad+\alpha_{n, p-2} \gamma\left\|e_{n, p-1}^{\prime \prime}\right\|+\left\|e_{n, p-2}^{\prime \prime}\right\| \\
& \quad+\alpha_{n, p-2} \alpha_{n, p-1} \gamma^{2}\left\|r_{n, p}\right\|+\alpha_{n, p-2} \gamma\left\|r_{n, p-1}\right\|+\left\|r_{n, p-2}\right\| \\
& \quad+\left(\alpha_{n, p-2} \gamma+\alpha_{n, p-2} \alpha_{n, p-1} \gamma^{2}\right) \Omega \\
& \quad+\left(\beta_{n, p-2}+\alpha_{n, p-2} \beta_{n, p-1} \gamma+\alpha_{n, p-2} \alpha_{n, p-1} \beta_{n, p} \gamma^{2}\right) \Omega .
\end{aligned}
$$

Continuing in the same way, we obtain

$$
\begin{aligned}
& \left\|v_{n, 1}-z\right\| \\
& \leq\left(1-\alpha_{n, 2}-\beta_{n, 2}+\alpha_{n, 2}\left(1-\alpha_{n, 3}-\beta_{n, 3}\right) \gamma+\alpha_{n, 2} \alpha_{n, 3}\left(1-\alpha_{n, 4}-\beta_{n, 4}\right) \gamma^{2}\right. \\
& \left.\quad+\cdots+\prod_{i=2}^{p-1} \alpha_{n, i}\left(1-\alpha_{n, p}-\beta_{n, p}\right) \gamma^{p-2}+\prod_{i=2}^{p} \alpha_{n, i} \gamma^{p-2} L \vartheta\right)\left\|z_{n}-z\right\| \\
& \quad+\alpha_{n, 2}\left\|e_{n, 2}^{\prime}\right\|+\alpha_{n, 2} \alpha_{n, 3} \gamma\left\|e_{n, 3}^{\prime}\right\|+\alpha_{n, 2} \alpha_{n, 3} \alpha_{n, 4} \gamma^{2}\left\|e_{n, 4}^{\prime}\right\| \\
& \quad+\ldots+\prod_{i=2}^{p} \alpha_{n, i} \gamma^{p-2}\left\|e_{n, p}^{\prime}\right\|+\prod_{i=2}^{p} \alpha_{n, i} \gamma^{p-2} L \vartheta b_{n}+\left\|e_{n, 2}^{\prime \prime}\right\|+\alpha_{n, 2} \gamma\left\|e_{n, 3}^{\prime \prime}\right\| \\
& \quad+\alpha_{n, 2} \alpha_{n, 3} \gamma^{2}\left\|e_{n, 4}^{\prime \prime}\right\|+\cdots+\prod_{i=2}^{p-1} \alpha_{n, i} \gamma^{p-2}\left\|e_{n, p}^{\prime \prime}\right\|
\end{aligned}
$$




$$
\begin{aligned}
& +\left\|r_{n, 2}\right\|+\alpha_{n, 2} \gamma\left\|r_{n, 3}\right\|+\alpha_{n, 2} \alpha_{n, 3} \gamma^{2}\left\|r_{n, 4}\right\|+\cdots+\prod_{i=2}^{p-1} \alpha_{n, i} \gamma^{p-2}\left\|r_{n, p}\right\| \\
& +\left(\alpha_{n, 2} \gamma+\alpha_{n, 2} \alpha_{n, 3} \gamma^{2}+\alpha_{n, 2} \alpha_{n, 3} \alpha_{n, 4} \gamma^{3}+\cdots+\prod_{i=2}^{p-1} \alpha_{n, i} \gamma^{p-2}\right) \Omega \\
& +\left(\beta_{n, 2}+\alpha_{n, 2} \beta_{n, 3} \gamma+\alpha_{n, 2} \alpha_{n, 3} \beta_{n, 4} \gamma^{2}+\cdots+\prod_{i=2}^{p-1} \alpha_{n, i} \beta_{n, p} \gamma^{p-2}\right) \Omega .
\end{aligned}
$$

By (6.3) and (6.11), we have

$$
\begin{aligned}
& \left\|z_{n+1}-z\right\| \\
& \leq\left(1-\alpha_{n, 1}-\beta_{n, 1}+\alpha_{n, 1}\left(1-\alpha_{n, 2}-\beta_{n, 2}\right) \gamma+\alpha_{n, 1} \alpha_{n, 2}\left(1-\alpha_{n, 3}-\beta_{n, 3}\right) \gamma^{2}\right. \\
& \left.+\cdots+\prod_{i=1}^{p-1} \alpha_{n, i}\left(1-\alpha_{n, p}-\beta_{n, p}\right) \gamma^{p-1}+\prod_{i=1}^{p} \alpha_{n, i} \gamma^{p-1} L \vartheta\right)\left\|z_{n}-z\right\| \\
& +\alpha_{n, 1}\left\|e_{n, 1}^{\prime}\right\|+\alpha_{n, 1} \alpha_{n, 2} \gamma\left\|e_{n, 2}^{\prime}\right\|+\alpha_{n, 1} \alpha_{n, 2} \alpha_{n, 3} \gamma^{2}\left\|e_{n, 3}^{\prime}\right\| \\
& +\cdots+\prod_{i=1}^{p} \alpha_{n, i} \gamma^{p-1}\left\|e_{n, p}^{\prime}\right\|+\prod_{i=1}^{p} \alpha_{n, i} \gamma^{p-1} L \vartheta b_{n}+\left\|e_{n, 1}^{\prime \prime}\right\|+\alpha_{n, 1} \gamma\left\|e_{n, 2}^{\prime \prime}\right\| \\
& +\alpha_{n, 1} \alpha_{n, 2} \gamma^{2}\left\|e_{n, 3}^{\prime \prime}\right\|+\cdots+\prod_{i=1}^{p-1} \alpha_{n, i} \gamma^{p-1}\left\|e_{n, p}^{\prime \prime}\right\| \\
& +\left\|r_{n, 1}\right\|+\alpha_{n, 1} \gamma\left\|r_{n, 2}\right\|+\alpha_{n, 1} \alpha_{n, 2} \gamma^{2}\left\|r_{n, 3}\right\|+\ldots+\prod_{i=1}^{p-1} \alpha_{n, i} \gamma^{p-1}\left\|r_{n, p}\right\| \\
& +\left(\alpha_{n, 1} \gamma+\alpha_{n, 1} \alpha_{n, 2} \gamma^{2}+\alpha_{n, 1} \alpha_{n, 2} \alpha_{n, 3} \gamma^{3}+\cdots+\prod_{i=1}^{p-1} \alpha_{n, i} \gamma^{p-1}\right) \Omega \\
& +\left(\beta_{n, 1}+\alpha_{n, 1} \beta_{n, 2} \gamma+\alpha_{n, 1} \alpha_{n, 2} \beta_{n, 3} \gamma^{2}+\cdots+\prod_{i=1}^{p-1} \alpha_{n, i} \beta_{n, p} \gamma^{p-1}\right) \Omega \\
& \leq\left(1-(1-L \vartheta) \prod_{i=1}^{p} \alpha_{n, i} \gamma^{p-1}\right)\left\|z_{n}-z\right\|+\sum_{i=1}^{p} \prod_{j=1}^{i} \alpha_{n, j} \gamma^{i-1}\left\|e_{n, i}^{\prime}\right\| \\
& +\prod_{i=1}^{p} \alpha_{n, i} \gamma^{p-1} L \vartheta b_{n}+\sum_{i=2}^{p} \prod_{j=1}^{i-1} \alpha_{n, j} \gamma^{i-1}\left\|e_{n, i}^{\prime \prime}\right\|+\sum_{i=2}^{p} \prod_{j=1}^{i-1} \alpha_{n, j} \gamma^{i-1}\left\|r_{n, i}\right\| \\
& +\left\|e_{n, 1}^{\prime \prime}\right\|+\left\|r_{n, 1}\right\|+\left(\sum_{i=2}^{p} \prod_{j=1}^{i-1} \alpha_{n, j} \gamma^{i-1}+\sum_{i=2}^{p} \prod_{j=1}^{i-1} \alpha_{n, j} \beta_{n, i} \gamma^{i-1}+\beta_{n, 1}\right) \Omega \\
& \leq\left(1-(1-L \vartheta) \prod_{i=1}^{p} \alpha_{n, i} \gamma^{p-1}\right)\left\|z_{n}-z\right\|
\end{aligned}
$$

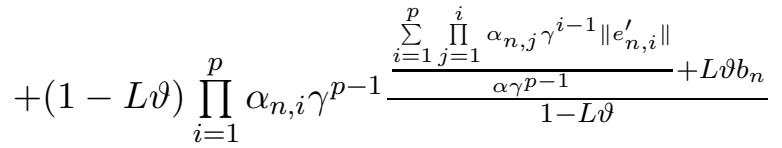

$$
\begin{aligned}
& +\sum_{i=2}^{p} \prod_{j=1}^{i-1} \alpha_{n, j} \gamma^{i-1}\left\|e_{n, i}^{\prime \prime}\right\|+\sum_{i=2}^{p} \prod_{j=1}^{i-1} \alpha_{n, j} \gamma^{i-1}\left\|r_{n, i}\right\|+\left\|e_{n, 1}^{\prime \prime}\right\|+\left\|r_{n, 1}\right\| \\
& +\left(\sum_{i=2}^{p} \prod_{j=1}^{i-1} \alpha_{n, j} \gamma^{i-1}+\sum_{i=2}^{p} \prod_{j=1}^{i-1} \alpha_{n, j} \beta_{n, i} \gamma^{i-1}+\beta_{n, 1}\right) \Omega \text {. }
\end{aligned}
$$


If $L \geq 1$ then from the assumption $L \omega<1$, where $\omega$ is the same as in (3.9), we have

$$
L \varsigma+\sqrt{1-2 \varrho+\nu^{2}}+L \sqrt{(1-2 \rho \delta) \tau^{2}+\rho^{2} \sigma^{2}}<1,
$$

whence we derive that $L \vartheta<1$. If $L<1$, then $L \vartheta<1$. By using (4.3), we note that all the conditions of Lemma 4.7 hold, and so, (6.12) and Lemma 4.7 guarantee that the sequence $\left\{z_{n}\right\}_{n=0}^{\infty}$ generated by Algorithm 5.3 converges strongly to a unique solution $z \in \mathcal{H}$ of problem (5.1).

Remark 6.2. Theorem 6.1 improves and generalizes Theorem 5.1 in [14].

\section{Extended General Projection Dynamical Systems}

In this section, we consider the dynamical system technique to study the existence and uniqueness of a solution of the extended general nonlinear quasi-variational inequality (2.1). Dupuis and Nagurney [4] introduced and studied the projected dynamical systems associated with variational inequalities, in which the right hand side of the ordinary differential equations is a projection operator. The novel feature of the projected dynamical system is that its set of stationary points corresponds of the set of the corresponding set of the solutions of the variational inequality problem. Thus the equilibrium and nonlinear programming problems, which can be formulated in the setting of the variational inequalities, can now be studied in the more general framework of the dynamical systems. It has been shown $[3,4,5,12,24,25,26]$ that these dynamical systems are useful in developing efficient and powerful numerical techniques for solving variational inequalities. In Section 3, we have already seen that the extended general nonlinear quasi-variational inequality (2.1) is equivalent to a fixed-point problem. We use this equivalence to suggest and analyze a projection dynamical system associated with (2.1). The fixed point formulation (3.1) enables us to suggest the following dynamical system

$$
\frac{d u}{d t}=\lambda\left[P_{K(u)}(g(u)-\rho T(u))-h(u)\right], \quad u\left(t_{0}\right)=u_{0} \in \mathcal{H},
$$

associated with the extended general nonlinear quasi-variational inequality (2.1), where $\lambda>0$ is a constant. The dynamical system (7.1) is called the extended general projection dynamical system associated with (2.1). Here the right hand is related to the projection and is discontinuous on the boundary. It is clear from the definition that a solution of (7.1) always stays in the constraint set. This implies that the qualitative results such as the existence, uniqueness, and continuous dependence of the solution on the given date (7.1), can be studied. The dynamical system describes the adjustment processes which may produce important transient phenomena prior to the achievement of a steady state. 
If $K(u) \equiv K$, then $P_{K(u)} \equiv P_{K}$ is the projection of $\mathcal{H}$ onto the closed and convex set $K$. In this case, the projection dynamical system (7.1) reduces to the following system:

$$
\frac{d u}{d t}=\lambda\left[P_{K}(g(u)-\rho T(u))-h(u)\right], \quad u\left(t_{0}\right)=u_{0} \in \mathcal{H}
$$

It is called extended general projection dynamical system associated with (2.2) and appears to be a new one.

If $K(u) \equiv K$ and $h \equiv I$ the identity operator, then the system (7.1) collapses to the following system:

$$
\frac{d u}{d t}=\lambda\left[P_{K}(g(u)-\rho T(u))-u\right], \quad u\left(t_{0}\right)=u_{0} \in \mathcal{H},
$$

which is studied in [17].

We need the following well-known concepts.

Definition 7.1. [24]. A dynamical system is said to be converge to the solution set $\Omega^{*}$ of (2.1) if, irrespective of the initial point, the trajectory of the dynamical system satisfies

$$
\lim _{t \rightarrow \infty} \operatorname{dist}\left(u(t), \Omega^{*}\right)=0
$$

where $\operatorname{dist}\left(u(t), \Omega^{*}\right)=\inf _{v \in \Omega^{*}}\|u-v\|$.

It is easy to see that if the set $\Omega^{*}$ consists only a point $u^{*}$, then (7.3) implies that $\lim _{t \rightarrow \infty} u(t)=u^{*}$.

If the dynamical system is still stable at $u^{*}$ in the Lyapunov sense, then the dynamical system is globally asymptotically stable at $u^{*}$.

Definition 7.2. [24]. A dynamical system is said to be globally exponentially stable with degree $\eta$ at $u^{*}$ if, irrespective of the initial point, the trajectory of the dynamical system satisfies

$$
\left\|u(t)-u^{*}\right\| \leq c_{0}\left\|u\left(t_{0}\right)-u^{*}\right\| \exp \left(-\eta\left(t-t_{0}\right)\right), \quad \text { for all } t \geq t_{0},
$$

where $c_{0}$ and $\eta$ are positive constants independent of the initial point. It is evident that globally exponentially stability is necessarily globally asymptotically stable and the dynamical system converges arbitrarily fast.

Lemma 7.1. [11]. Let $\hat{u}$ and $\hat{v}$ be real-valued nonnegative continuous functions with domain $\left\{t: t \geq t_{0}\right\}$ and let $\alpha(t)=\alpha_{0}\left(\left|t-t_{0}\right|\right)$, where $\alpha_{0}$ is a monotone increasing function. If for all $t \geq t_{0}$,

$$
\hat{u}(t) \leq \alpha(t)+\int_{t_{0}}^{t} \hat{u}(s) \hat{v}(s) d(s),
$$


then

$$
\hat{u}(t) \leq \alpha(t)+\exp \left\{\int_{t_{0}}^{t} \hat{v}(s) d(s)\right\} .
$$

By using Lemma 7.1 and Theorem 3.2, we establish the existence of a unique solution of the extended general projection dynamical system (7.1) associated with the extended general quasi-variational inequality (2.1).

Theorem 7.2. Let $T, g, h$ and $\rho$ be the same as in Theorem 3.2 and let all the conditions of Theorem 3.2 hold. Then for each $u_{0} \in \mathcal{H}$, there exists a unique continuous solution $u(t)$ of the extended general projection dynamical system (7.1) with $u\left(t_{0}\right)=u_{0}$ over $\left[t_{0}, \infty\right)$.

Proof. According to Theorem 3.2, Problem (2.1) has a unique solution $u^{*} \in \mathcal{H}$ with $h\left(u^{*}\right) \in K\left(u^{*}\right)$. Hence, it follows Lemma 3.1 that $h\left(u^{*}\right)=P_{K\left(u^{*}\right)}\left(g\left(u^{*}\right)-\right.$ $\left.\rho T\left(u^{*}\right)\right)$. Define

$$
F(u)=\lambda\left\{P_{K(u)}(g(u)-\rho T(u))-h(u)\right\}, \quad \forall u \in \mathcal{H},
$$

where $\lambda>0$ is a constant. Then for all $u, v \in \mathcal{H}$, we have

$$
\begin{aligned}
& \|F(u)-F(v)\| \\
\leq & \lambda\left(\left\|P_{K(u)}(g(u)-\rho T(u))-P_{K(v)}(g(v)-\rho T(v))\right\|+\|h(u)-h(v)\|\right) \\
\leq & \lambda(\|u-v\|+\|u-v-(h(u)-h(v))\| \\
& +\left\|P_{K(u)}(g(u)-\rho T(u))-P_{K(v)}(g(u)-\rho T(u))\right\| \\
& \left.+\left\|P_{K(v)}(g(u)-\rho T(u))-P_{K(v)}(g(v)-\rho T(v))\right\|\right) \\
\leq & \lambda(\|u-v\|+\|u-v-(h(u)-h(v))\|+\varsigma\|u-v\| \\
& +\|g(u)-g(v)-\rho(T(u)-T(v))\|) .
\end{aligned}
$$

Since $T$ is $\delta$-strongly monotone with respect to $g$ and $\sigma$-Lipschitz continuous, $g$ is $\tau$ Lipschitz continuous, $h$ is $\varrho$-strongly monotone and $\nu$-Lipschitz continuous, in a similar way to that of proofs of (3.6) and (3.7), we get

$$
\|u-v-(h(u)-h(v))\| \leq \sqrt{1-2 \varrho+\nu^{2}}\|u-v\|
$$

and

$$
\|g(u)-g(v)-\rho(T(u)-T(v))\| \leq \sqrt{(1-2 \varrho \delta) \tau^{2}+\rho^{2} \sigma^{2}}\|u-v\| .
$$

Combining (7.4)-(7.6), we conclude that

$$
\|F(u)-F(v)\| \leq \lambda(1+\omega)\|u-v\|,
$$


where $\omega$ is the same as in (3.9). Accordingly, the operator $F$ is locally Lipschitz continuous on $\mathcal{H}$. Hence, for each $u_{0} \in \mathcal{H}$, there exists a unique and continuous solution $u(t)$ of the extended general projection dynamical system (7.1), defined on an interval $t_{0} \leq t<\mathcal{T}$ with the initial condition $u\left(t_{0}\right)=u_{0}$. Let $\left[t_{0}, \mathcal{T}\right)$ be its maximal interval of existence, then we show that $\mathcal{T}=\infty$. For any $u \in \mathcal{H}$, we have

$$
\begin{aligned}
\|F(u)\|= & \lambda\left\|P_{K(u)}(g(u)-\rho T(u))-h(u)\right\| \\
\leq & \lambda\left(\left\|P_{K(u)}(g(u)-\rho T(u))-h\left(u^{*}\right)\right\|+\left\|h(u)-h\left(u^{*}\right)\right\|\right) \\
\leq & \lambda\left(\left\|u-u^{*}\right\|+\left\|u-u^{*}-\left(h(u)-h\left(u^{*}\right)\right)\right\|\right. \\
& \left.+\left\|P_{K(u)}(g(u)-\rho T(u))-P_{K\left(u^{*}\right)}\left(g\left(u^{*}\right)-\rho T\left(u^{*}\right)\right)\right\|\right) \\
= & \lambda\left(\left\|u-u^{*}\right\|+\left\|u-u^{*}-\left(h(u)-h\left(u^{*}\right)\right)\right\|\right. \\
& +\left\|P_{K(u)}(g(u)-\rho T(u))-P_{K\left(u^{*}\right)}(g(u)-\rho T(u))\right\| \\
& \left.+\left\|P_{K\left(u^{*}\right)}(g(u)-\rho T(u))-P_{K\left(u^{*}\right)}\left(g\left(u^{*}\right)-\rho T\left(u^{*}\right)\right)\right\|\right) \\
\leq & \lambda\left(\left\|u-u^{*}\right\|+\left\|u-u^{*}-\left(h(u)-h\left(u^{*}\right)\right)\right\|+\varsigma\left\|u-u^{*}\right\|\right. \\
& \left.+\left\|g(u)-g\left(u^{*}\right)-\rho\left(T(u)-T\left(u^{*}\right)\right)\right\|\right) \\
\leq & \lambda(1+\omega)\left\|u-u^{*}\right\| \\
\leq & \lambda(1+\omega)\left\|u^{*}\right\|+\lambda(1+\omega)\|u\|,
\end{aligned}
$$

then,

$$
\|u(t)\| \leq\left\|u_{0}\right\|+\int_{t_{0}}^{t}\|F(u(s))\| d s \leq\left(\left\|u_{0}\right\|+k_{1}\left(t-t_{0}\right)\right)+k_{2} \int_{t_{0}}^{t}\|u(s)\| d s,
$$

where $k_{1}=\lambda(1+\omega)\left\|u^{*}\right\|$ and $k_{2}=\lambda(1+\omega)$. Therefore, using Lemma 7.1, we have

$$
\|u(t)\| \leq\left(\left\|u_{0}\right\|+k_{1}\left(t-t_{0}\right)\right) e^{k_{2}\left(t-t_{0}\right)}, \quad t \in\left[t_{0}, \mathcal{T}\right) .
$$

Hence, the solution is bounded for $t \in\left[t_{0}, \mathcal{T}\right)$ if $\mathcal{T}$ is finite. Thus, $\mathcal{T}=\infty$.

By using the technique of Xia and Wang [23, 24], we show that the trajectory of a solution of the extended general projection dynamical system (7.1) converges to a unique solution of the extended general nonlinear quasi-variational inequality (2.1).

Theorem 7.3. Let $T, g, h$ and $\rho$ be the same as in Theorem 3.2 and let all the conditions of Theorem 3.2 hold. If $1-\mu<\varrho-\varsigma<\tau$, where $\mu$ is the same as in (3.3), then the extended general projection dynamical system (7.1) converges globally exponentially to a unique solution of the extended general nonlinear quasi-variational inequality (2.1). 
Proof. Since all the conditions of Theorem 3.2 hold, Theorem 3.2 guarantees the existence of a unique solution $u^{*} \in \mathcal{H}$ with $h\left(u^{*}\right) \in K\left(u^{*}\right)$ for problem (2.1). Therefore, Lemma 3.1 implies that $h\left(u^{*}\right)=P_{K\left(u^{*}\right)}\left(g\left(u^{*}\right)-\rho T\left(u^{*}\right)\right)$. On the other hand, in view of Theorem 7.2, the extended general projection dynamical system (7.1) has a unique solution $u(t)$ over $\left[t_{0}, \mathcal{T}\right)$ for any fixed $u_{0} \in \mathcal{H}$. Let $u(t)=u\left(t, t_{0}: u_{0}\right)$ be the solution of (7.1) with $u\left(t_{0}\right)=u_{0}$. We consider the Lyapunov function $L$ defined on $\mathcal{H}$ as follows:

$$
L(u)=\left\|u-u^{*}\right\|^{2}, \quad u \in \mathcal{H} .
$$

Then from (7.1), (7.7) and $\varrho$-strongly monotonicity of $h$, it follows that

$$
\begin{aligned}
\frac{d L}{d t}= & \frac{d L}{d u} \frac{d u}{d t}=2\left\langle u(t)-u^{*}, \frac{d u}{d t}\right\rangle \\
= & 2 \lambda\left\langle u(t)-u^{*}, P_{K(u)}(g(u)-\rho T(u))-h(u)\right\rangle \\
= & -2 \lambda\left\langle u(t)-u^{*}, h(u)-h\left(u^{*}\right)\right\rangle \\
& +2 \lambda\left\langle u(t)-u^{*}, P_{K(u)}(g(u)-\rho T(u))-h\left(u^{*}\right)\right\rangle \\
\leq & -2 \lambda \varrho\left\|u(t)-u^{*}\right\|^{2}+2 \lambda\left\|u(t)-u^{*}\right\| \\
& \quad\left\|P_{K(u)}(g(u)-\rho T(u))-P_{K\left(u^{*}\right)}\left(g\left(u^{*}\right)-\rho T\left(u^{*}\right)\right)\right\| .
\end{aligned}
$$

Since $T$ is $\delta$-strongly monotone with respect to $g$ and $\sigma$-Lipschitz continuous, $g$ is $\tau$-Lipschitz continuous, by using the condition (3.2), we have

$$
\begin{aligned}
\| & P_{K(u)}(g(u)-\rho T(u))-P_{K\left(u^{*}\right)}\left(g\left(u^{*}\right)-\rho T\left(u^{*}\right)\right) \| \\
\leq & \left\|P_{K(u)}(g(u)-\rho T(u))-P_{K\left(u^{*}\right)}(g(u)-\rho T(u))\right\| \\
& +\left\|P_{K\left(u^{*}\right)}(g(u)-\rho T(u))-P_{K\left(u^{*}\right)}\left(g\left(u^{*}\right)-\rho T\left(u^{*}\right)\right)\right\| \\
\leq & \varsigma u-u^{*}\|+\| g(u)-g\left(u^{*}\right)-\rho\left(T(u)-T\left(u^{*}\right)\right) \| \\
\leq & \left(\varsigma+\sqrt{(1-2 \rho \delta) \tau^{2}+\rho^{2} \sigma^{2}}\right)\left\|u-u^{*}\right\| .
\end{aligned}
$$

Substituting (7.9) in (7.8), we have

$$
\begin{aligned}
\frac{d}{d t}\left\|u(t)-u^{*}\right\|^{2} & \leq-2 \lambda\left(\varrho-\varsigma-\sqrt{(1-2 \rho \delta) \tau^{2}+\rho^{2} \sigma^{2}}\right)\left\|u(t)-u^{*}\right\|^{2} \\
& =-2 \lambda \gamma\left\|u(t)-u^{*}\right\|^{2},
\end{aligned}
$$

where $\gamma=\varrho-\varsigma-\sqrt{(1-2 \rho \delta) \tau^{2}+\rho^{2} \sigma^{2}}$. Thus, we have

$$
\left\|u(t)-u^{*}\right\| \leq\left\|u(t)-u^{*}\right\| e^{-\lambda \gamma\left(t-t_{0}\right)}
$$

The condition (3.3) and the fact that $1-\mu<\varrho-\varsigma<\tau$ guarantee that $\gamma>0$. So the trajectory of the solution of the extended general projection dynamical system (7.1) global exponentially converges to a unique solution of the extended general nonlinear quasi-variational inequality (2.1). 


\section{REFERENCES}

1. C. Baiocchi and A. Capelo, Variational and Quasivariational Inequalities, New York, 1984.

2. A. Bensoussan and J. L. Lions, Application des Inéquations Variationelles en Control et en Stochastiques, Dunod, Paris, 1978.

3. J. Dong, D. Zhang and A. Nagurney, A projected dynamical systems model of general financial equilibrium with stability analysis, Math. Comput. Modell., 24(2) (1996), 3544.

4. P. Dupuis and A. Nagurney, Dynamical systems and variational inequalities, Ann. Oper. Res., 44 (1993), 19-42.

5. T. L. Friesz, D. H. Bernstein and R. Stough, Dynamical systems, variationl inequalities and control theoretical models for predicting time-varying unban network flows, Trans. Sci., 30 (1996), 14-31.

6. K. Goebel and W. A. Kirk, A fixed point theorem for asymptotically nonexpansive mappings, Proc. Amer. Math. Soc., 35 (1972), 171-174.

7. G. Isac, Complementarity Problems, Lecture Notes in Math., No. 1528, Springer-Verlag, New York, Berlin, 1992.

8. W. A. Kirk and H. K. Xu, Asymptotic pointwise contractions, Nonlinear Anal., 69 (2008), 4706-4712.

9. A. S. Kravchuk and P. J. Neittaanmaki, Variational and Quasi Variational Inequalities in Mechanics, Springer, Dordrecht, Holland, 2007.

10. L. S. Liu, Ishikawa and Mann iterative process with errors for nonlinear strongly accretive mappings in Banach spaces, J. Math. Anal. Appl., 194 (1995), 114-125.

11. R. K. Miller and A. N. Michel, Ordinary Differential Equations, Academic Press, New York, 1982.

12. A. Nagurney and D. Zhang, Projected Dynamical Systems and Variational Inequalities with Applications, Kluwer Academic Publishers, Dordrecht, Holland, 1995.

13. M. A. Noor, Existence results for quasi-variational inequalities, Banach J. Math. Anal., 1 (2007), 186-194.

14. M. A. Noor, On general quasi-variational inequalities, Journal of King Saud UniversityScience, 24 (2012), 81-88.

15. M. A. Noor, Resolvent dynamical systems for mixed variational inequalities, Korean $J$. Comput. Appl. Math., 9 (2002), 15-26.

16. M. A. Noor and Z. Huang, Three-step iterative methods for nonexpansive mappings and variational inequalities, Appl. Math. Comput., 187 (2007), 680-685.

17. M. A. Noor, K. I. Noor and H. Yaqoob, On general mixed variational inequalities, Acta Appl. Math., 110(1) (2010), 227-246. 
18. X. Qin and M. A. Noor, General Wiener-Hopf equation technique for nonexpansive mappings and general variational inequalities in Hilbert spaces, Appl. Math. Comput., 201 (2008), 716-722.

19. D. R. Sahu, Fixed points of demicontinuous nearly Lipschitzian mappings in Banach spaces, Comment. Math. Univ. Carolin, 46(4) (2005), 653-666.

20. P. Shi, Equivalence of variational inequalities with Wiener-Hopf equations, Proc. Amer. Math. Soc., 111 (1991), 339-346.

21. A. H. Siddiqi and Q. H. Ansari, An algorithm for a class of quasivariational inequalities, J. Math. Anal. Appl., 145 (1990), 413-418.

22. A. H. Siddiqi and Q. H. Ansari, Strongly nonlinear quasivariational inequalities, J. Math. Anal. Appl., 149 (1990), 444-450.

23. Y. S. Xia, On convergence conditions of an extended projection neural network, Neural Comput., 17 (2005), 515-525.

24. Y. S. Xia and J. Wang, A recurrent neural network for solving linear projection equations, Neural Network, 137 (2000), 337-350.

25. Y. S. Xia and J. Wang, On the stability of globally projected dynamical systems, $J$. Optim. Theory Appl., 106 (2000), 129-150.

26. D. Zhang and A. Nagurney, On the stability of the projected dynamical systems, $J$. Optim. Theory Appl., 85 (1985), 97-124.

Qamrul Hasan Ansari

Department of Mathematics

Aligarh Muslim University

Aligarh 202 002, India

and

Department of Mathematics \& Statistics

King Fahd University of Petroleum \& Minerals

Dhahran, Saudi Arabia

E-mail: qhansari@gmail.com

Javad Balooee

Department of Mathematics, Sari Branch

Islamic Azad University

Sari, Iran

E-mail: javad.balooee@gmail.com

Jen-Chih Yao

Center for Fundamental Science

Kaohsiung Medical University

Kaohsiung 80708, Taiwan

E-mail: yaojc@cc.kmu.edu.tw 